615668800

\title{
DEVELOPMENT OF AN INTERNET-BASED SYNCHRONOUS GIS FOR COLLABORATIVE SPATIAL DECISION MAKING
}

\author{
By \\ Lijun GU \\ B.Sc., \\ China University of Geosciences \\ Wuhan, P.R.China, 1994 \\ A thesis \\ Presented to Ryerson University \\ in partial fulfillment of the \\ requirement for the degree of \\ Master of Applied Science \\ in the Program of \\ Civil Engineering
}

Toronto, Ontario, Canada, 2004

(C)(Lijun Gu) 2004 
UMI Number: EC53464

\section{INFORMATION TO USERS}

The quality of this reproduction is dependent upon the quality of the copy submitted. Broken or indistinct print, colored or poor quality illustrations and photographs, print bleed-through, substandard margins, and improper alignment can adversely affect reproduction.

In the unlikely event that the author did not send a complete manuscript and there are missing pages, these will be noted. Also, if unauthorized copyright material had to be removed, a note will indicate the deletion.

\section{UMI}

UMI Microform EC53464

Copyright 2009 by ProQuest LLC

All rights reserved. This microform edition is protected against unauthorized copying under Title 17, United States Code.

ProQuest LLC

789 East Eisenhower Parkway

P.O. Box 1346

Ann Arbor, MI 48106-1346 


\section{AUTHOR'S DECLARATION}

I hereby declare that I am the sole author of this thesis.

I authorize Ryerson University to lend this thesis to other institutions or individuals for the purpose of scholarly research.

\section{Lijun $\mathrm{Gu}$}

Department of Civil Engineering

Ryerson University

I further authorize Ryerson University to reproduce this thesis by photocopying or by other means, in total or in part, at the request of other institutions or individuals for the purpose of scholarly research.

Lijun Gu

Department of Civil Engineering

Ryerson University 


\section{BORROWER'S PAGE}

Ryerson University requires the signatures of all persons using or photocopying this thesis. Please sign below, and give address and date. 


\title{
Development of An Internet-Based Synchronous GIS for Collaborative Spatial Decision Making
}

\author{
Master of Applied Science, 2004, Lijun Gu \\ Department of Civil Engineering \\ Ryerson University
}

\begin{abstract}
Urban and transportation development largely depends on innovative information technologies for decision making support in its planning and management processes to achieve beneficial economic, social and environmental outcomes. Among these technologies, techniques and tools for collaborative visualization, manipulation, and exploration of spatial information are particularly useful. Existing Geographic Information Systems (GISs), however, lack of the capability to support collaborative spatial decision making (CSDM). This thesis presents a research effort in the development of GIS software tools that support synchronized collaboration between multiple participants via the Internet, to explore urban and transportation development scenarios for collective decision making. While the design and development focused on integrating decision making tools with commercial GIS development Toolkits (e.g., MapObjects Java Edition) using collaborative Java APIs, the approach and insights gained should be of general interest. The initial usefulness testing indicates that an Internet-based synchronous GIS can help improve decision making processes of urban corridor planning and ease participation in such decision making activities.
\end{abstract}




\section{ACKNOWLEDGEMENTS}

Over the last two years, this thesis was completed with the efforts, guidance, patience and encouragement of many persons to whom I want to express my sincere thanks. While it would be impossible to acknowledge all of them, it is my sincere pleasure to extend special thanks to the following individuals:

First, my very special thanks go to my supervisor, Dr. Songnian $\mathrm{Li}$, for his continuous and patient criticism, concern and valuable scientific remarks and guidance, especially his understanding, friendship, and effort in keeping my research on the right track.

I would also like to extend my sincere thanks to Dr. Jonathan Li and Dr. Liping Fang for their insightful suggestions on my research and their acting as the members of my thesis examining committee.

Thanks are also going to all my fellow students at Ryerson University for their direct and indirect help and support on my research and studies.

Finally, my special thanks to my wife, Fan Ding who has sacrificed so much for this adventure and whose complete support and encouragement have greatly helped make this thesis a success, and to whom I owe too much to return.

The financial support provided by Dr. Songnian $\mathrm{Li}$ and graduate scholarship received from Ryerson University has made this research possible and it has been greatly appreciated. 


\section{TABLE OF CONTENTS}

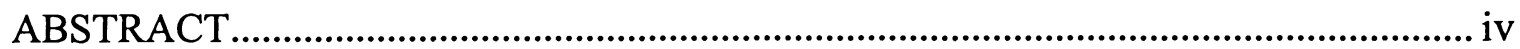

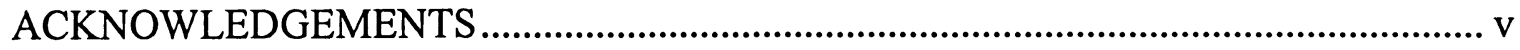

TABLE OF CONTENTS .................................................................................. vi

LIST OF FIGURES ..................................................................................... viii

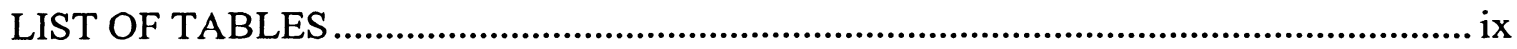

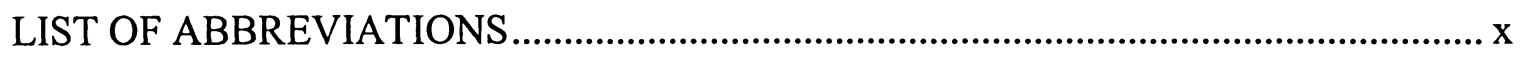

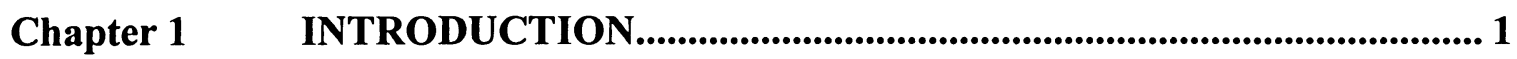

1.1 RESEARCH CONTEXT ............................................................................... 1

1.2 RESEARCH OBJECTIVES ......................................................................... 5

1.3 SIGNIFICANCE AND CONTRIBUTIONS.............................................................. 6

1.4 RESEARCH LIMITATIONS........................................................................ 7

1.5 ORGANIZATION OF THE THESIS ................................................................ 8

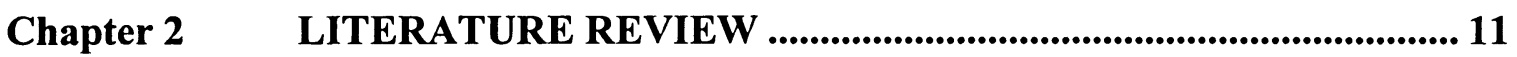

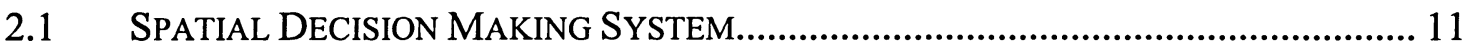

2.1.1 Spatial Decision Support System (SDSS)....................................... 11

2.1.2 GIS vs. SDSS ....................................................................... 12

2.1.3 Collaborative Spatial Decision Making (CSDM) ................................... 13

2.2 COMPUTER-SUPPORTED COOPERATIVE WORK (CSCW) AND GROUPWARE ...... 15

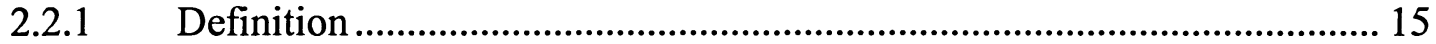

2.2.2 Groupware Classification.................................................................. 16

2.2.3 Display-sharing and Event-sharing................................................. 17

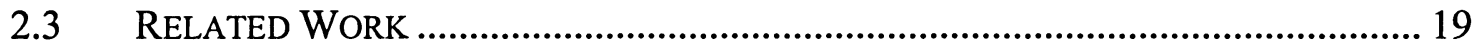

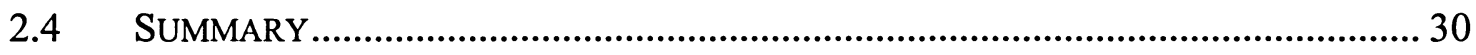

Chapter 3 COMPARATIVE STUDY OF SELECTED COLLABORATIVE

FRAMEWORKS......................................................................................................... 32

3.1 TANGO INTERACTIVE..................................................................... 33

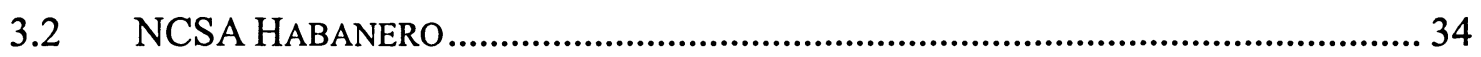

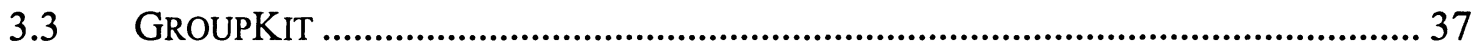

3.4 ShaStra Web Based Collaborative SubSTRate ....................................... 39

EVALUATION AND SUMMARY ................................................................ 41

Chapter 4 FRAMEWORK DESIGN OF A COLLABORATIVE GIS........... 44

4.1 SYSTEM REQUIREMENTS ................................................................... 45

4.2 FUNCTIONAL REQUIREMENTS ................................................................ 46

4.3 ARCHITECTURE DESIGN OF COLLABORATIVE WORKSPACE ............................. 48 
4.3.1 Session Management ……...................................................................... 50

4.3.2 Communication among Collaborating Clients.............................................. 51

4.3.3 Floor Control........................................................................................... 51

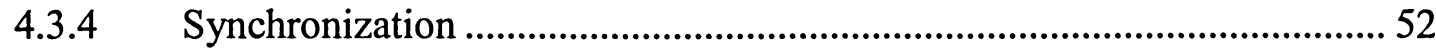

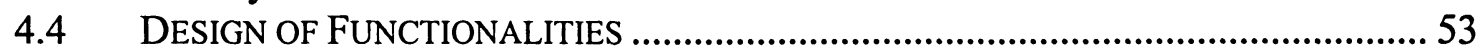

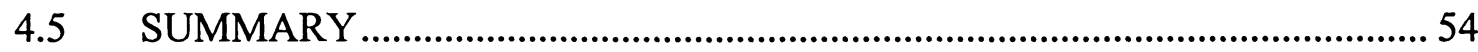

Chapter 5 PROTOTYPE DEVELOPMENT ........................................................... 55

5.1 PRototyPE ARCHITECTURE AND DEVELOPING TOOLS.....................................55

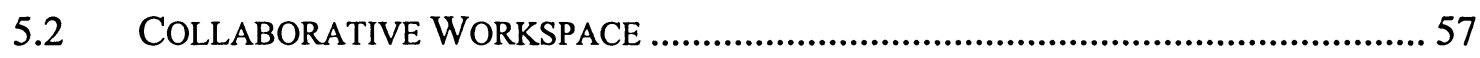

5.2.1 Server Program ...................................................................................... 58

5.2.2 Client Program ............................................................................................ 59

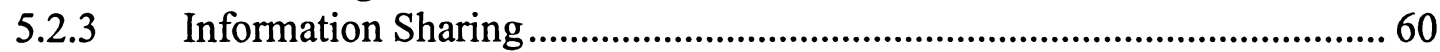

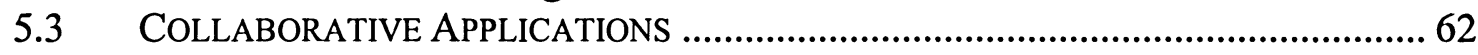

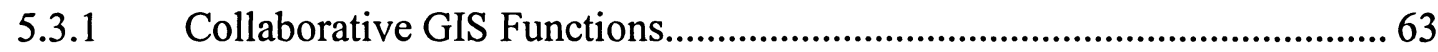

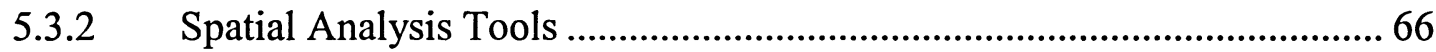

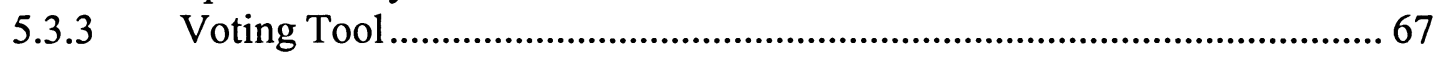

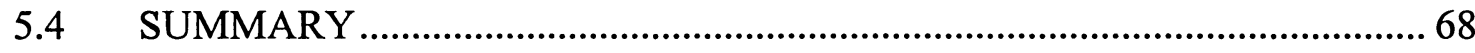

Chapter 6 CGIS APPLICATION IN CORRIDOR PLANNING...................... 69

6.1 AN OVERVIEW Of TRANSPORTATION CORRIDOR PlanNing Process ............... 69

6.2 A TASK MODEL OF CORRIDOR PLANNING DECISION MAKING............................ 71

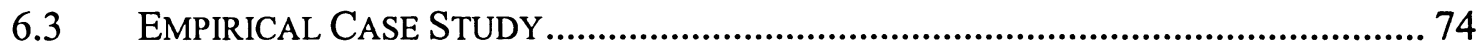

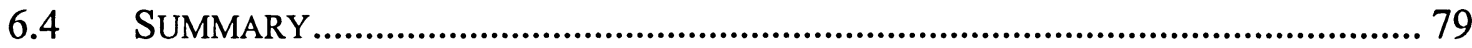

Chapter 7 CONCLUSIONS AND RECOMMENDATIONS............................... 80

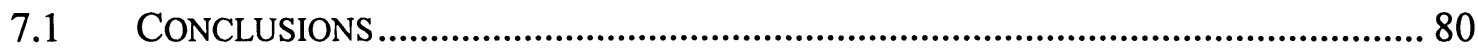

7.2 RECOMMENDATIONS FOR FUTURE RESEARCH .................................................. 84

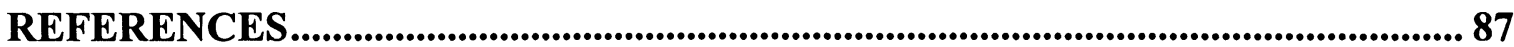




\section{LIST OF FIGURES}

Figure 2.1 SDSS Architecture (Hall et al., 1997) ……..................................................... 12

Figure 3.1 Architecture of TANGO Interactive System (Podgorny et al., 1998) ............. 33

Figure 3.2 GroupKit Architecture (Roseman and Greenberg, 1992).................................. 38

Figure 3.3 Shastra Runtime Architecture (Anupam and Bajaj, 1993).............................. 40

Figure 4.1 Hardware Architecture Model of Collaborative Framework ............................ 48

Figure 4.2 Software design architecture ............................................................................... 49

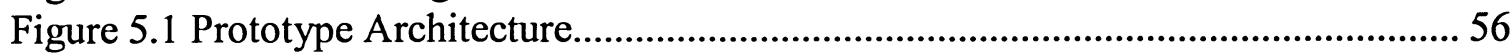

Figure 5.2 Information flow between server and client..................................................... 58

Figure 5.3 GUI of Session Status (Create, Join and Leave) ...............................................6 60

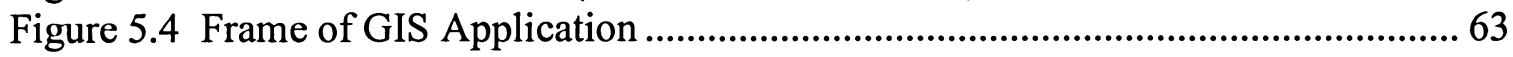

Figure 5.5 In the same session, spatial data loaded into one map canvas will also be loaded into other map canvas synchronously ................................................................6 65

Figure 5.6 Screen shot of collaborative GIS module.......................................................... 66

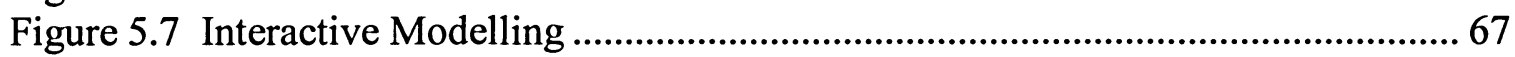

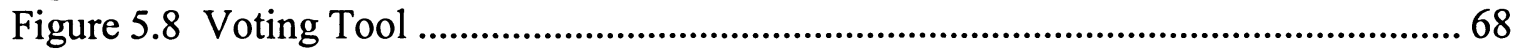

Figure 6.1 A task model for collaborative GIS technology in support of corridor planning (After Nyerges and Jankowski, 2001b) ....................................................................... 73

Figure 6.2 Study Area (contain 4 layers: Roads, Water, Vegetation and Land Use) ...... 75

Figure 6.3 Four Alternatives and the affected features...................................................... 76

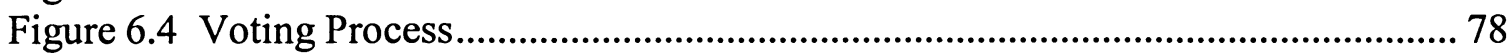




\section{LIST OF TABLES}

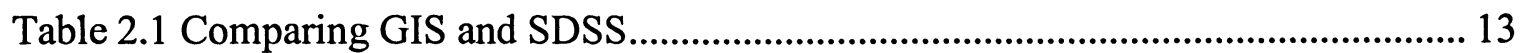

Table 2.2 Time/Space Classification Matrix (Finley, 1997)............................................... 16

Table 3.1 Comparison of Collaborative Frameworks.................................................. 42

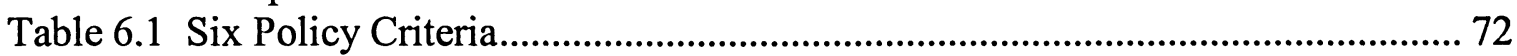

Table 6.2 Ten Technical Criteria ………..................................................................... 72 


\section{LIST OF ABBREVIATIONS}

$\begin{array}{ll}\text { API: } & \text { Application Programming Interface } \\ \text { CAD: } & \text { Computer-aided Design } \\ \text { CDSM: } & \text { Collaborative Spatial Decision Making } \\ \text { CGIS: } & \text { Collaborative GIS } \\ \text { CPS: } & \text { Collaborative Planning System } \\ \text { CSCW: } & \text { Computer-Supported Cooperative Work } \\ \text { DSS: } & \text { Decision Support System } \\ \text { ESRI: } & \text { Environment Source Research Institute } \\ \text { GDSS: } & \text { Group Decision Support System } \\ \text { GIS: } & \text { Geographic Information System } \\ \text { GSDS: } & \text { Group Spatial Decision Support } \\ \text { GUI: } & \text { Graphical User Interface } \\ \text { HTTP: } & \text { Hyper Text Transfer Protocol } \\ \text { JDK: } & \text { Java Develop Kit } \\ \text { JPEG: } & \text { Joint Photographic Expert Group } \\ \text { MCD: } & \text { Multi-criteria Decision } \\ \text { MCDM: } & \text { Multi-criteria Decision Making } \\ \text { NCSA: } & \text { National Center for Supercomputing Applications } \\ \text { NPAC: } & \text { Northeast Parallel Architecture Centre } \\ \text { PPGIS: } & \text { Public Participation GIS } \\ \text { SDSS: } & \text { Spatial Decision Support Systems } \\ \text { SQL: } & \text { Structured Query Language } \\ \text { TCP/IP: } & \text { Transmission Control Protocol/Internet Protocol } \\ \text { TI: } & \text { TANGO Interactive } \\ \text { TIFF: } & \text { Tag Image File Format } \\ \text { UML: } & \text { Unified Modeling Language } \\ \text { WWW: } & \text { Word Wide Web } \\ & \end{array}$




\section{Chapter 1}

\section{INTRODUCTION}

Geographic Information System (GIS) is a multi-disciplinary activity and typically a GIS project will involve people from many backgrounds and organizations. For example, urban and transportation development largely depends on innovative information technologies required for decision making support in its planning and management processes to achieve beneficial economic, social and environmental outcomes. Among these technologies, techniques and tools for collaborative visualization, manipulation, and exploration of spatial information are particularly useful, especially in modern democratic planning and management processes that require input from a large group of diverse stakeholders including the public. The importance of such collaboration is clear and much work has been accomplished to enable organizations to combine their resources and to cooperate on the collection and analysis of decision related data as well as the ability to share resources and data. It is also necessary to support groups of people from different disciplines and in different locations in discussing and making decisions about GIS related topics (Armstrong, 1994).

\subsection{Research Context}

Spatial decision making is linked to location and associated with spatial relationships. Many spatial decision problems are wicked because they contain intangibles that cannot 
be easily quantified and modeled, their structure is partially known or burdened by uncertainty. These problems require the participation and collaboration of people representing diverse areas of competence, political agendas and social interests. As a consequence, diverse groups must often be involved to generate solutions to pervasive spatial problems (Jankowski and Nyerges, 2001a).

Spatial decision making that deals with geographic problems has been around for quite a long time. However, the interest in group participatory decision making has only been growing as more and more people realize that those who concern about environmental, land use, natural resource, and transportation issues and those who are affected by decisions should be part of the process. Many geographic decision problems are viewed as unstructured and their solutions demand the participation of multiple stakeholders with varying interests and values.

Current trend in modern organizations towards flatter structures and the involvement of many stakeholder groups in solving decision problems have created a need for information technology capable of supporting participatory spatial decision making. Such information technology has been developed in recent years, resulting in computerized group decision making support systems which aim solving spatial decision problems, such as transportation corridor planning, site selection, land use and development, choice of environmental and economic strategies, and urban/regional development. 
This surge of interest in collaborative spatial decision making (CDSM) has been spurred not only by the trend in business organizations, but also foremost by realizing that effective solution to spatial decision problems requires collaboration and consensus building.

The need for computerized decision support results from the importance of group decision making and problem solving carried out during meetings. Despite the negative characteristics (e.g., getting off the subject, too lengthy, inconclusive, disorganized, no goals or agenda, redundant or digressive discussion) (Mosvick and Nelson, 1987), the attractiveness of a group approach to decision making comes in general from the fact that individual contributions are increased by a synergistic effect resulting from meeting dynamics. Jankowski and Nyerges (2001b) identified several human decision making abilities that information technology might augment in meeting. These include:

1. helping decision makers formulate, frame, or assess decision situations by identifying the salient features of the environment, recognizing needs, identifying appropriate objectives by which to measure the successful resolution of an issue;

2. providing support in enhancing the abilities of decision makers to obtain and analyze possible impacts of alternative courses of action; and

3. enhancing the ability of decision makers to interpret impacts in terms of objectives, leading to an evaluation of alternatives and selection of preferred alternative option. 
Consequently, a final outcome of a computer-supported decision meeting can be more than a simple sum of individual contributions. The attractiveness of a computer-supported group approach to spatial decision making comes from a possibility of engaging diverse participants as competent stakeholders through computer-mediated communication, problem exploration, and negotiation support.

Spatial decision making problems commonly involve three categories of participants: stakeholders, decision makers, and technical specialists (Jankowski and Nyerges, 2001b). The diversity of participant categories may include a range of expertise levels in virtually any decision problem - from novice through intermediate to expert. Reducing the complexity of a decision problem by reducing the cognitive workload of participants is one goal of developing collaborative decision support system (Armstrong, 1993). Reducing cognitive workload will hopefully lead to a more thorough treatment of information, by exposing initial assumption more clearly, facilitating critiques of the accuracy of information, and subsequently resulting in more effective and equitable participatory decisions.

In the past decade, GIS and its offspring spatial decision support systems (SDSS) were suggested as information technology aids to facilitate understanding of spatial problems and facilitate computer-supported spatial decision making (Amtrong, 1993). It has been argued that GIS is the core technology in SDSS, but specialized techniques are needed to support spatial decision making (Densham, 1991). However, currently GIS and SDSS are not designed to support groups, but many applications involve groups. Armstrong (1993) 
proposed several suggestions for extending the development of GIS to support locational problem solving oriented to groups. Reitsma (1996) provides insight into the structure and support of decision making using a group-based SDSS for water resource management. New developments in information technology focusing on the Internet and the Word Wide Web (WWW or the Web) have provided new possibilities for better access to spatial information and enhanced benefits for its use.

While the mainstream GIS technology concentrates on the creation of easy-to-use, ubiquitous mapping and spatial analysis tools, existing GISs, however, do not have tools to support interactions among group members such as shared graphics, group modeling, and group consensus building tools, and have limited capability to support collaborative spatial decision making (CSDM). To address this issue, an Internet-based synchronous GIS for collaborative spatial decision making has been developed in this research.

\subsection{Research Objectives}

The aim of this research is to develop an Internet-based synchronous GIS prototype for collaborative spatial decision making. In particular, this research:

1. investigates several existing collaborative frameworks and selects one of them as a reference framework for this research.

2. designs an Internet-based synchronous GIS framework which includes collaborative workspace architecture and collaborative function modules. 
3. develops a workable collaborative workspace prototype and collaborative applications that can facilitate the computer-supported decision making of a small group, which includes exploring spatial and attribute data, performing spatial analysis, articulating and sharing decision criteria, evaluating the alternatives, and negotiating the consensus solutions.

4. tests the performance of this collaborative GIS prototype in a case study of transportation corridor planning.

\subsection{Significance and Contributions}

This research makes the following contributions to the overall field of knowledge in this area of collaborative spatial decision support systems:

- It presents a different approach of developing an Internet-based synchronous GIS that supports collaborative spatial decision making.

- It develops a collaborative GIS application with the latest GIS developing tool ESRI MapObjects Java Edition which greatly improves the development efficiency.

- The collaborative workspace is developed to provide a useful framework for other developers to design and develop collaborative GIS applications and other similar applications that support group decision making.

- The developed research prototype demonstrates the feasibility of integrating CSCW (groupware), spatial decision making and GIS capabilities at both 
component and system levels onto the Internet infrastructure to support collaborative spatial decision making.

- The result obtained from performance testing provides initial measure of the efficiency the proposed solution brings to group spatial decision making. The documentation and procedures of the research prototype will enable further research work.

\subsection{Research Limitations}

The research reported in this thesis is subject to several constraints and limitations listed as follows:

- Collaborative Framework Selection: Due to the research budget and license availability, it is not feasible to check all existing collaborative software in the market. In this research, I only investigated four freely available collaborative software packages.

- GIS Data Format: Data format incompatibilities not only hinder sharing data transparency, but also affect the design and implementation of collaborative workspace and applications (Li, 2003). The data format supported by MapObjects APIs includes shapefile, image file such as JEPG and TIFF, computer-aided design (CAD) (DGN, DXF, and DWG). Since usually the size of image files is large and $\mathrm{CAD}$ files are not geo-referenced, when programming the GIS application, only data in shapefile format were used in this research. 
- Software Functionality: In developing the collaborative workspace based on the designed framework, the software functionality is limited because of the time limits, this could potentially affect the overall performance of the collaborative GIS prototype.

- Hardware: The hardware configurations and availability were constrained by the research budget. No hardware alternatives were available for testing the collaborative GIS in different hardware environments. Therefore, the result of performance testing may be affected by certain hardware configuration.

- Internet Access: In this research, the required Internet access for the collaborative GIS development and testing was obtained through the civil engineering department network which is connected to the Ryerson University's fibre optic backbone. The prototype testing was performed under the Geomatics lab environment.

\subsection{Organization of the Thesis}

This thesis is organized into seven chapters, starting with this chapter which presents the overall introduction of research context, research motivations and objectives, research significance and contributions, and limitations of the research that may affect the research result.

The second chapter aims at presenting in-depth literature review of collaborative GIS and necessary background. It covers the related research in the field of collaborative GIS for 
spatial decision making from the prospects of different time/place arrangements. It also introduces the knowledge of spatial decision support system (SDSS), collaborative spatial decision making (CSDM), computer-supported cooperative work (CSCW) and groupware, and the theory of GIS-supported collaborative decision making.

Chapter 3 extensively investigates four research related collaborative frameworks, focusing on describing and analyzing those collaborative frameworks' architecture, collaboration scheme, and implementation and user development. Among those frameworks, NCSA Habanero was selected as the reference collaborative framework in this research.

Chapter 4 describes the framework design of collaborative GIS. It starts with the system and functional analysis and requirement of the Internet-based synchronous GIS for collaborative spatial decision making, then proposes the architecture design of collaborative environment and functionalities design of collaborative applications that support spatial decision making.

Chapter 5 presents the development of the research prototype system, with a detailed introduction of collaborative workspace and prototyped collaborative functional modules. The collaborative functions are developed at three levels: collaborative mapping tools, collaborative spatial analysis tools and group decision making tool. 
Chapter 6 explores how the collaborative GIS prototype could be used in transportation corridor planning with a case study.

Chapter 7 summarizes the conclusions of the research and proposes some recommendations for further research. 


\section{Chapter 2}

\section{LITERATURE REVIEW}

\subsection{Spatial Decision Making System}

\subsubsection{Spatial Decision Support System (SDSS)}

The concept of decision support system (DSS) is based on the seminal work by Simon and Associates in 1950s and 1960s (Armstrong and Densham, 1990). Sprague (1980) defined Decision Support Systems (DSSs) as computer systems that are: (1) designed to solve semi- and un-structured problems that upper level managers often face; (2) able to combine analytical models with traditional data storage and retrieval functions; (3) userfriendly and accessible by decision makers with minimal computer experience; and (4) flexible and adaptable to different decision making approaches. Extending this definition, Armstrong et al. (1986) used the term Spatial Decision Support Systems (SDSS) to refer to computer programs that assist decision makers generate and evaluate alternative solutions to semi-structured spatial problems through the integration of analytical models, spatial data and traditional geoprocessing software (such as GIS).

Armstrong and Densham (1990) suggest that five key components are needed in a SDSS, including Database Management, Analysis Logic (objectives, constraints, and decision rules), Spatial Display, Report Generation and User's Interface. Proposed by Hall et al. (1997), the basic architecture of SDSS could be represented in such a manner as illustrated in Figure 2.1. 


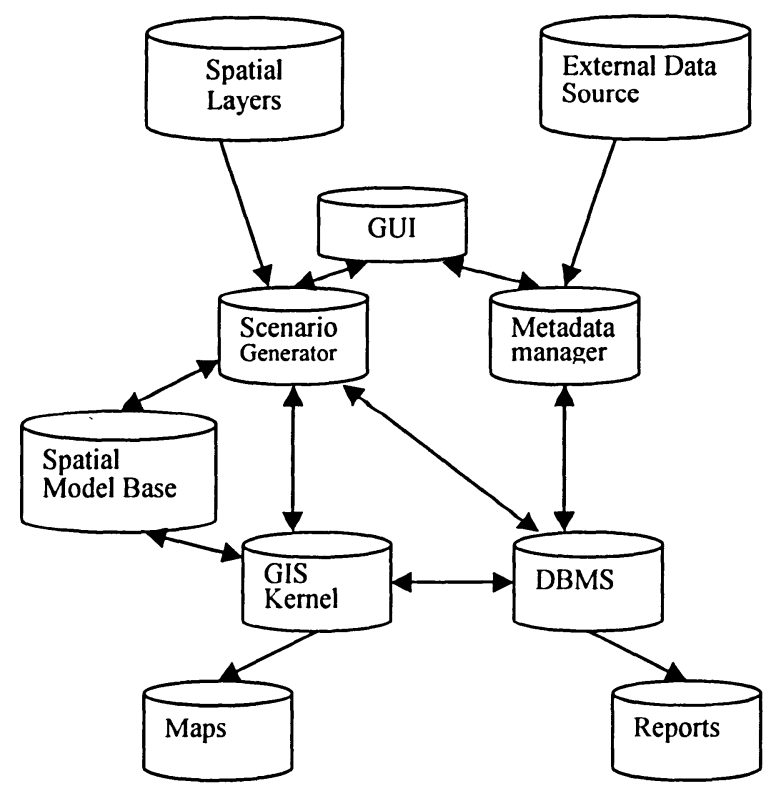

Figure 2.1 SDSS Architecture (Hall et al., 1997)

\subsubsection{GIS vs. SDSS}

Though a GIS can be used in a decision making process, its performance cannot be expected to be as effective as that of a SDSS because 1) insufficient attention is given to the process and context in which decisions are made; and 2) most GIS provide only capabilities for map analysis and do not support the domain specific analytical and statistical modeling required by many decision makers. These shortcomings of GIS have led to the development of SDSS, which are explicitly meant for spatial problem solving. Thus, SDSS evolved from GIS and has been developed explicitly to accommodate the context and the chosen process of spatial decision making. Table 2.1 demonstrates the major differences between SDSS and GIS. 
Table 2.1 Comparing GIS and SDSS

\begin{tabular}{|l|l|l|}
\hline $\begin{array}{c}\text { The contents of } \\
\text { comparison }\end{array}$ & \multicolumn{1}{|c|}{ GIS } & \multicolumn{1}{c|}{ SDSS } \\
\hline Focus & Information & Decision making \\
\hline Object oriented & Well-defined problems & Ill-defined problems \\
\hline Function & $\begin{array}{l}\text { Describe the real world and } \\
\text { detect the problems }\end{array}$ & $\begin{array}{l}\text { Support decision making in } \\
\text { order to exploit and remodel } \\
\text { the real world }\end{array}$ \\
\hline Characteristics & Processing routinely, inflexible & $\begin{array}{l}\text { Processing according to } \\
\text { requirements, flexible }\end{array}$ \\
\hline Result output & Maps and tables & $\begin{array}{l}\text { Decision making schemes } \\
\text { and best solution }\end{array}$ \\
\hline Status of user & Positive, system-driven & Active, user-driven \\
\hline $\begin{array}{l}\text { Scope of } \\
\text { application }\end{array}$ & Management at middle level & $\begin{array}{l}\text { Decision making at high } \\
\text { level }\end{array}$ \\
\hline Objective & $\begin{array}{l}\text { Improve working efficiency, } \\
\text { seek for quickness }\end{array}$ & $\begin{array}{l}\text { Improve decision making } \\
\text { ability, seek for effectiveness }\end{array}$ \\
\hline
\end{tabular}

\subsubsection{Collaborative Spatial Decision Making (CSDM)}

The inability of GIS technology to adequately represent dynamic spatial systems is well documented. GIS software packages lack adequate spatial modeling capabilities, spatial analytical tools, spatio-temporal data structures to represent such systems (Bennett, 1995). Spatial decision support systems (SDSS) overcome some of these issues by incorporating modeling and analytical tools needed to address domain specific problems (Densham, 1991). However, these systems do not possess the model base management 
capabilities needed to support the simulation of processes operating over different spatiotemporal scales. Furthermore, both GIS and SDSS suffer from what Armstrong (1993) refers to as the "GIS bottleneck" that limits the utility of these technologies in collaborative decision making environments.

In recent years, facilitating public participation in decision making concerning spatial issues has created a need for information technology capable of supporting collaborative spatial decision making. The CSDM, or Group Spatial Decision Making, has been one of the most important topics in GIS community. A CSDM is an interactive computer-based system that facilitates the solution of ill-structured problems by a number of decision makers who work together as a team. The main objective of a CSDM is to augment the effectiveness of decision groups through the interactive sharing of information between the group members and the computer (Karacapilidis and Pappis, 1997).

There are four different arrangements of CSDM: (1) the same location and time (collaborative work using a conferencing room with a local area computer network), (2) the same location and different time (collaborative work using leave-behind word processing supported by a local, or wide area computer network), (3) different locations and same time (collaborative work using interactive desktop audio and video, supported by a wide area network, a dedicated wide band-width telephone line, or a satellite link), and (4) different locations and different times (collaborative work using e-mail, wide area network, and network-resident multimedia tools). 


\subsection{Computer-Supported Cooperative Work (CSCW) and Groupware}

\subsubsection{Definition}

The origin of CSCW can be traced back to the early 1960 when Engelbart envisioned enhancing human intellect through technologically medicated and facilitated formal collaboration (Baecker, 1993a). Greif and Cashman first introduced the phrase “Computer-Supported Cooperative Work" to describe Engelbart's concepts (Grudin, 1994). In 1978, Peter and Trudy introduced the term "groupware" to encompass those tools (Gruidin, 1994). Groupware gained popularity in the late 1980s as media awareness and usage of the term increased. Since the advance of the Internet, some groupware tools, such as email, ftp, real-time computer conferencing, computer teleconferencing, desktop videoconferencing, bulletin boards, group schedules, screen-sharing software and electronic classroom, have been available. Scientific American (1990) defines CSCW as "Technology which allows people in remote places to interact with each other and with the same documents and files through voice, data and video links".

Groupware is an enabling technology for such environments and reflects a fundamental change in emphasis from using computers to solve problems to using computers to facilitate human interaction (Ellis et al., 1991). Baecker (1993a) defined Groupware as "information technology used to help people work together more effectively". The principle functions of groupware are information sharing, document authoring, messaging, computer conferencing, group calendars, project management, and support for team building (Zwass, 1998). 


\subsubsection{Groupware Classification}

Finley (1997) classified groupware according to two primary dimensions based on place and time. Place refers to the locations of participants using the groupware product. Both face-to-face meeting and virtual meetings are supported and referred to as same-place and different-place meetings, respectively. Time refers to synchronous communication (realtime applications such as spoken conversation) or asynchronous communication (where interaction takes place over an extended time frame). Table 2.2 provides a synopsis of the possible combinations of time and place.

Table 2.2 Time/Space Classification Matrix (Finley, 1997)

\begin{tabular}{||l|l|l||}
\hline \multirow{1}{*}{ Same Place } & \multicolumn{1}{|c|}{ Same Time } & \multicolumn{1}{c|}{ Different Time } \\
& Face-to-face interaction & Asynchronous interaction \\
& - conversation & - bulletin board postings \\
& - meeting facilitation & - presentation \\
& - group decision support & - project management \\
& systems room & - team room \\
\hline \hline Different Place & Synchronous distributed & Asynchronous distributed \\
& interaction & interaction \\
& - phone conversation & - voice mail \\
& - conference calls & - email \\
& - shared screen interfaces & - collaborative writing \\
\hline
\end{tabular}

Synchronous groupware systems run in real-time and support group communication and collaboration using such techniques as instant messaging. An example is an electronic meeting system used for brainstorming. In asynchronous systems, users access stored 
messages or send messages to be viewed at a later time, such as email. Synchronous and asynchronous approaches affect communication behaviour differently. For example, in asynchronous systems, communications tend to be lengthy with multiple, simultaneous discussion threads, while in synchronous systems the participants tend to focus on a single topic at one time. A different kind of distinction between synchronous and asynchronous systems is given by Udell et al. (2001) who differentiate between systems not just on the basis of the temporal characteristics of communications, but also on how tasks and information are shared.

A model of groupware that has frequently been used to represent both synchronous and asynchronous systems is the CSCW framework, developed by Dix and Beale (1996). The model distinguishes between participants in the collaborative process, and explicitly emphasizes the need to develop a joint understanding of the problem by the participants. A well-known type of groupware is the Group Decision Support System (GDSS) which is used to facilitate such group processes as brainstorming, reaching consensus by voting, surveying experts, and negotiation where parties resolve conflicting interests by communication (Zwass, 1998). This not only saves time because of the parallel processing it allows, but also permits more ideas to be presented then in a traditional face-to-face meeting.

\subsubsection{Display-sharing and Event-sharing}

In the context of general taxonomy of synchronous collaborating systems, current systems fall into two broad categories: display-sharing and event-sharing architectures. 
In display-sharing architecture, there is only one instance of the application running on a single machine. The conference agent replicates user input and application display on all workstations participating in the session. Shared display systems replicate entire functionality of the application used for collaboration. A typical example of such system is Microsoft's NetMeeting. Display-sharing mechanism uses a naive approach to collaboratory. The advantage is simplicity of implementation. This technology turns every stand-alone application into a collaborative version outweighing all deficiencies. This technology has some disadvantages such as from the functional point of view, there is no flexibility in shaping collaboratory process, and from the perspective of performance, the entire display information must be passed to all collaborators, which requires significant effort to compress the transmitted data stream. Transmission errors lead to inconsistent state that can manifest itself in grotesque fashion. Display-sharing technology could be applied in remote administration of computer systems.

Event-sharing mechanism exchanges state-changing events over the network. Each participating workstation runs a local copy of the application. This architecture allows application designed for a single individual to be used by a group of people. In this architecture, all events could be shared. Such architecture is typically implemented by modifying the window toolkit so that the events are trapped before being sent to local applications and distributed to other session participants. The typical example systems use this architecture are Tango Interactive and NCSA Habanero.

Event-sharing architecture is flexible, generates minimal network traffic, can implement security at any required level, can support session playback and recording, and allows 
inclusion of intelligent agents enhancing system functionality. The overall architecture of event sharing may better fit the distributed Internet paradigm.

\subsection{Related Work}

Geographic research and decision making are group activities more than ever before. Behind development and implementation of GIS over the years, one of the key expected applications has been its potential to support collaborative activities (e.g., complex location-based decision making) with integration of information representing multiple perspectives and disciplines (MacEahren, 2000). However, the methods and tools of cartography and GIS have been developed for use by individuals.

Existing GISs are not well suited to support collaborative activities such as decision making. GISs are designed for application to well structured problems, it lacks tools that support decision making directly (Armstrong, 1994). SDSS address these issues by extending GIS with tools designed to overcome these limitations, individually in most initial efforts (Armstrong, 1993). The NCGIA Initiative 17 on Collaborative Spatial Decision making served as an important stimulus for research directed to addressing the issues collectively (Densham et al., 1995).

Within Geographic Information Science, there is a growing volume of literature on group work with geospatial information (MacEachren, 2000, 2001). Much of the attention has focused on group spatial decision support (GSDS) (an outgrowth of earlier work in 
SDSS), with the focus on the design of environments that merge GIS with multi-criteria evaluation methods and decision support tools (Armstrong, 1993; Jankowski and Nyerges, 2001). While substantial progress has been made in SDSS and GDSS (Jankowski and Nyerges, 2001), limited attention has been given to collaboration focusing on graphic displays. Thus generally the research fails to address many issues that are likely to be critical for collaboration using the standard tools of GIS.

A lot of GIS research have investigated distributed spatial collaboration. Rinner (1999) realizes the need to support group discussions through linking individual contributions to map elements. His combination of "argumentation theory" and geographical representations presents a novel approach for asynchronous spatial planning.

The Centre for Computational Geography at the University of Leeds has taken a different approach toward distributed spatial collaboration. They are investigating the idea of increasing public participation in democracy through web-based GIS (Evans et al., 1999). Many governments provide information on the web for the public, but spatial information has been restricted to simply exploration and no response technique. As a result, they conducted a test-bed project that had local people contribute to town improvement ideas online. This work offers a limited solution with respect to our vision of spatial collaboration. They have explored a way for the public to provide anonymous and spatial feedback to their government, while we are interested in more intricate scenarios of people solving spatial problems together using synchronous communication. 
Research focused on collaboration about geographic phenomena and problems can be approached from many perspectives. From the perspective of GIS as mediator of humanhuman collaboration, the most useful categorization of collaborative situations is one that emphasizes spatial and temporal differences in the collaborative setting (MacEahren, 2000). Spatially, the key distinction is between collaborators who are co-located (same place collaboration) and those located remotely (different place collaboration). Temporally, collaboration can be categorized as synchronous (same time) or asynchronous (different time). Armstrong (1993) and Shiffer (1998) proposed that collaboration can involve participants sharing work at the same or different place and the same or different time. For spatial collaboration the dynamic visual display becomes the mediator that supports collaboration in these four "meeting situations", particularly for those participants that are located at different places.

Research dealing with same place collaboration has focused on the social and organizational issues involved in group spatial decision making and on development and application of synchronous methods and tools to support spatial decision making, Shiffer $(1992 ; 1993 ; 1995)$ has developed some innovative tools to facilitate both public understanding of planning proposals and public involvement in planning meetings. The ideas have been implemented in Collaborative Planning System (CPS). The CPS links GIS with multimedia methods to increase the breadth of information forms and perspectives incorporated in decision making. This system enables an integration of gesture, visual, and verbal discourse. Jankowski and Nyerges (2001) have developed a 
systematic program of GIS-supported, same-time-same-place collaborative decision making system.

Recent advances in geographic information technology that support large distributed databases and on-line GIS have lead to several prototypes that facilitate different-place collaboration (Churcher and Churcher, 1999; Jones et al., 1997). Such efforts in GIS toward different-place collaboration are quite limited and have emphasized using webbased groupware integrated with WebGIS (Churcher and Churcher, 1999; Rinner, 2001).

Asynchronous different-place work has occurred for long history (e.g., through mail to exchange information and perspectives). Much of the initial work on different place collaboration has been directed to asynchronous sharing of and access to information, as well as software. However, specific attention has also been directed to methods and tools that support synchronous collaboration at different place.

In the work focusing on same-place synchronous design, Jankowski et al. (1997) presented a collaborative spatial decision support prototype "Spatial Group Choice" and applied it in habitat site selection. Spatial Group Choice has been developed to facilitate the computer-supported interactions of small-groups in face-to-face meeting environment. The system is comprised of two modules: a multi-criteria evaluation module (Group Choice) with a sub module for multi-criteria decision making (MCDM) and a voting sub module (Consensus), and a module for spatial visualization, customized from ArcView 2. Group Choice integrated with ArcView 2 (AV-2) based on a loose- 
coupling strategy. Decision makers can separately explore the habitat sites data in AV-2 module, select decision criteria in MCDM module, and perform evaluation to those criteria. The group can then vote in Consensus module to come up with to some consensus according to the criteria that are selected. After this step, the decision makers come back to MCDM module to clarify priorities, weigh the criteria, and perform sensitivity analysis. The decision maker can see the result displayed in AV-2 module. Finally, the group vote by submitting the individual site rankings to the Consensus module, and the voting result will be ranked and displayed.

Synchronous different-place work has been possible since the advent of the telegraph and telephone. The ability to exchange geospatial data, maps and imagery (as well as voice and video) is essential to making same time/different-place geocollaboration possible.

Churcher and Churcher $(1996 ; 1999)$ proposed GroupARC in their series papers. GroupARC is a lightweight GIS browser that provides a tool that can support people to collaboratively view, query and annotate spatial data together even though they are geographically separated. GroupARC has been developed using the freely available GroupKit toolkit. With this approach, each participant must have Internet access and also his/her own copy of the GroupKit and GroupARC software. GroupARC would be used in addition to, rather than instead of, a conventional GIS. GroupARC does not endeavor to carry out any computationally intensive analysis or topological functions itself. It is assumed that at least one participant will have access to a GIS to perform these tasks, leaving GroupARC as a vehicle for explaining and discussing the results of such analysis. 
Research dealing with the same place collaboration GIS can be further partitioned into two partially overlapping components in the context of the decision making. One involves research directed to group decision making by experts - Group-Spatial Decision Support Systems (Group-SDSS). The second is directed to group decision making that involves public participation - Public Participation GIS (PPGIS).

Most of the research directed to Group-SDSS has pursued an explicit or implicit goal of making better decision support tools (MacEahren, 2000). The typical approach is to develop a conceptual framework and associated methods that will extend existing GISs and SDSS environments to support group work. Armstrong $(1993,1994)$, in a pair of papers, identifies important links to ongoing research in $\mathrm{CSCW}$ and outlines a set of core objectives for Group-SDSS. Nyerges et al. (1997) develop a concept model of the decision making process involved in deciding among competing transportation improvement site selection projects. Their task model is then used to summarize the influence of various aspects of group-based GIS technology on decision making in this context. Nyerges and Jankowski (1997) propose a theoretical framework considering the human components of GIS-supported collaborative decision making. Their framework is more generally derived from a comprehensive assessment of research dealing with GIS use, collaboration, group decision making, and information technology more generally. This assessment yielded 21 "aspects" of the group decision making problem that the framework attempts to take into consideration. The framework has been applied to identifying questions related to the use of Group-SDSS . 
To the research directed to PPGIS, more emphasis has been given to the social and political processes that determine who has access to geospatial data and GISs and to the role of geographic information technologies in empowering or disenfranchising participants in public policy decisions. As Obermeyer (1998) points out, PPGIS is a direct outgrowth of research on societal implications of GISs.

Collaboration issues in PPGIS have been considered primarily from a practical rather than a theoretical perspective (Roche and Humeau, 1999). Healey (1996) describes that urban planning should be approached using collaborative interaction among stakeholders. Development and implementation of successful PPGIS technology requires a conceptual framework to approach both design of these technologies and assessment of their use (Howard, 1998; Craig and Elwood, 1998). Rinner (1997), in a series of papers that complement work on PPGIS, focuses on tools that facilitate the process of collaboration within CSDM, whether among specialists or the wider community. His approach integrated geospatial representations within a discussion forum environment that is used to structure argumentation processes. The approach emphasizes 'geographical mediation', defined as moderated discussion in a spatial planning procedure.

Comparing with Group-SDSS, PPGIS puts more focus on helping users learn about the problem context and following up decisions. Nyerges et al. (1997) delineated four stages in the collaborative public decision making process: preliminary assessment, problem definition, decision making and decision follow up. It is likely that different technologies or combinations of them will be appropriate for facilitating collaboration at each stage. 
Some work has also been carried out on development of visual tools to support collaborative decision making. Wood et al. (1997) proposed that the ideal collaborative systems should support both 'instructor-driven' collaborations and interaction among multiple independent participants. For the later, they suggest that the environment should support data exchange, shared control, dynamic interaction, ease of learning, and shared application modes. Brewer et al. (2000) and MacEachren et al. (1999) developed collaborative geovisualization tools to support group data exploration.

In the study that investigated the use of maps as mediators for group work with geospatial data, Jankowski and Nyerges (2001) found that maps were used primarily in the analytical integration phase of a decision making process. Armstrong and Densham (1995) presented some initial ideas about the role of cartographic representations as mediators for geocollaboration, in the context of group decision making specifically. They proposed a set of new map types designed to facilitate the process of making comparisons among alternative facility location scenarios, thus map that acted as shared objects to coordinate perspectives.

Among the few attempts to develop visual tools intended specifically to facilitate group work with geospatial information, Rinner $(1997,1999)$ focused attention on how to graphically mediate georeferenced discussions on shared objects, coordinate perspectives and actions within a planning context. He considers georeferenced discussions to have two primary components: (1) arguments (contained in messages) and (2) geographical 
objects (e.g., parcels, roads). Rinner's approach to enabling discussion incorporates mapbased graphics within a discussion that uses maps for sharing and storing information.

The visual tools designed to facilitate geocollaboration will require attention to the representations of participants and their actions. Such representation must be integrated with displays of the information that collaboration is about. These representations of participants must provide mechanisms to depict their own presence in the environment, their own actions on objects in the environment and toward other participants, other participant's presence in the environment; and other participant's deictic actions toward objects and the user (MacEachren et al., 1999).

The GeoVISTA Center at Penn State University has explored extending geovisualizations to support distributed, collaborative work (MacEachren et al., 2001). Geovisualizations, or Geographic Visualizations, use visual representations to make spatial contexts and problems visible to experts. Displaying these representations across multiple locations enables experts to share ideas about geographic spaces.

Pang and Fernandez (1995) describe a prototype of collaborative visualization environments --Real-Time Environmental Information Network and Analysis System (REINAS) that was designed to support analysis of geospatial data. This prototype system collected data from distributed instruments, stored information in a federated database, and supported collaborative visualization of oceanographic and meteorological information. Visualization capabilities were organized into three modes: monitor, forecast and analysis. Collaboration facilities included a session manager, mechanism to 
share data and data display tools, 'floor' control (to manage control among multiple participants), local and public windows, audio/video support, and different collaboration/compression levels. Related research by Wood and his colleagues (Wood et $a l ., 1996 ; 1997)$ provides a conceptual framework for developing system architecture to extend the single-user dataflow model of visualization toolkits to support multiple users.

For group work with geospatial information, it is needed to represent not only who is participating at what level, but also which representations of data they are using and what they are doing with those representations. The following are the most important overarching research challenges summarized by MacEachren et al. (2001):

- Develop a theoretical understanding of the cognitive and social aspects of both local and remote collaboration mediated through display objects in a geospatial context;

- Develop approaches to multi-user system interfaces that support group work;

- Understand the ways in which characteristics of methods and tools provided to support collaboration influence the outcome of group work.

- Initiate a parallel, concerted effort focused on integrating, implementing, and investigating the role of the visual, geospatial display in collaborative science, education, design, and group decision support;

Some research has been undertaken to improve understanding of traditional interaction with geospatial information. Cohen (1999) and McGee et al. (2000) studied collaborative interaction with paper maps for command and control operations. The specific goal was to improve the understanding of interaction with $2 \mathrm{D}$ static maps, so that this 
understanding could guide development and design of a voice, pen, and touch-based interface to a large screen display (McGee and Cohen, 2001; McGee et al., 2001).

The collaborative process has many dimensions. MacEachren (2003) identify six dimensions particularly important from the perspectives of both system design and evaluation. The first three dimensions focus on the human components of collaborative environment and include: problem context, collaboration tasks, and perspective commonality. The second three dimensions emphasize the computing infrastructure (e.g., networking and visualization) that can support collaboration, thus they are considered as system dimensions. These system dimensions include: spatial and temporal context, interaction characteristics, and tools to mediate group work.

McGrath (1984) delineated four general components within the process of group work: generate (ideas and options), negotiate, choose, and execute. Spatial decisions (e.g., land use planning, environmental management, and highway design) are generally contentious. Geocollaboration methods and tools for these applications must support shared understanding and resolution of disputes among different points of view.

Research associated with design and implementation of geospatial collaborative technologies is still at an early stage of development. Thus, there have been only limited empirical studies addressing the technologies and process of group spatial decision making designed to facilitate group work. 
Several investigations have been conducted to create or adapt technology that can facilitate collaborative use of geospatial data. Specific problems considered include: (a) representing information in group settings and allowing group members to interact with and change the representations; (b) adapting and applying electronic meeting software designed to facilitate both individual and collective decision making; and (c) facilitating efficient use of expert knowledge to assist non-experts in information retrieval (MacEahren, 2000).

MacEachren (2003) proposed that interaction among participants within a geocollaborative environment involves three interrelated factors. First is group size and aggregation (how many participants are collaborating?). Second is the topology of connections among group members or subgroups (who is connected to whom?). Third are constraints on form and flow of information among participants (what promotes or impedes information dissemination crucial to collaboration?)

\subsection{Summary}

Collaborative GIS is a relatively new but important area of research for geography and related disciplines. There is not much literature in this field. In the extended geocollaboration field: GroupSDSS and PPGIS, much research focuses on asynchronous collaboration. Only few studies addressed synchronous collaboration.

The dramatic changes of technology developments in the field of GIS, CSCW and groupware, Internet, teleconferencing, virtual reality, mobile communications systems 
and others will have a substantial impact on the merge of those technologies into collaborative GIS. In addition to the geospatial technology issues that must be addressed, and the range of human factors issues to be considered, geocollaborative technologies are likely to have profound impacts on our society. 


\section{Chapter 3}

\section{COMPARATIVE STUDY OF SELECTED}

\section{COLLABORATIVE FRAMEWORKS}

This chapter presents several collaborative frameworks from some notable research work focusing on describing and analyzing their collaborative frameworks' architecture, collaboration scheme, and implementation and user development.

The main objective of this stage is to select a suitable collaborative framework that could provide the platform to develop Internet-based collaborative applications. Since ESRI MapObjects Java Edition is a good developing tool to develop Java-based GIS applications, one key criterion to select the collaborative framework is the compatibility with ESRI MapObjects Java APIs. Due to the research budget, whether the framework could be freely obtained is also an important fact.

To reach the objective, the related collaborative frameworks were reviewed and some freely available collaborative frameworks were selected for study. Those free frameworks including source code of framework and APIs were obtained, installed and tested separately. After this step, those frameworks were evaluated according to their features including the type of APIs, which programming languages can be used to develop applications, whether the framework supports MapObjects APIs, whether the framework 
and its APIs can be freely available. After comprising those frameworks, the most suitable one was selected as the reference framework for this research.

\subsection{TANGO Interactive}

TANGO Interactive (TI) is a web-based synchronous collaborative system developed at the Northeast Parallel Architecture Centre (NPAC) at Syracuse University, the system provides multi-user collaborative environment.

TI system consists of three modules: Meeting Engine, Collaborative Client, and Application Server. Figure 3.1 shows the architecture of the system.

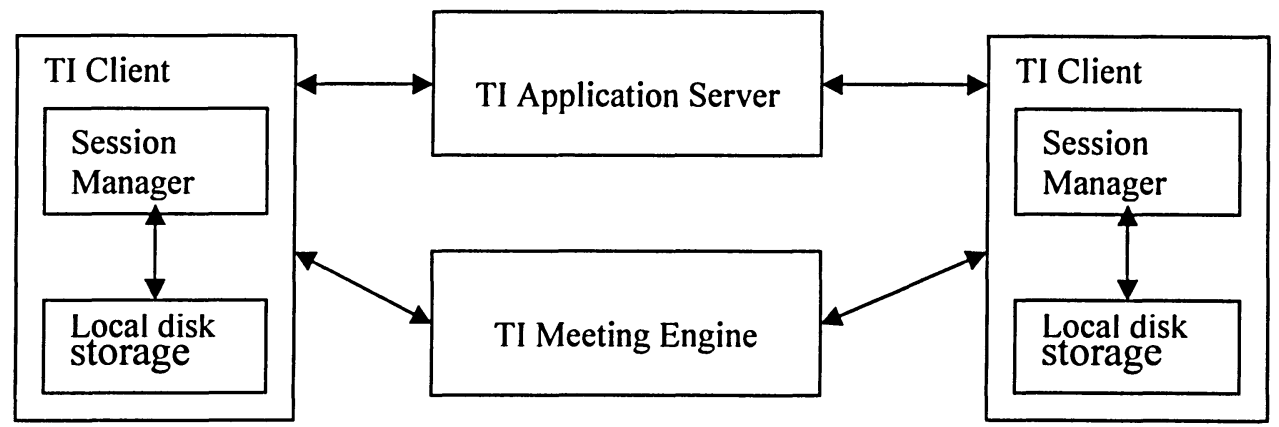

Figure 3.1 Architecture of TANGO Interactive System (Podgorny et al., 1998)

Meeting engine is the core component in TI system. It is not an HTTP server, it is more like an IP router (Podgorny et al., 1998). It makes sure the messages get to the intended receivers. Meeting engine communicates directly with the TI Session Managers. It maintains the dynamic state of the collaboration system such as established sessions and running applications. Meeting engine also provides communication channels for message 
flow in the system. Using these channels, the collaborative applications started by the active users can exchange data.

The TI Client package is virtually invisible to the end-user. Its most important function is to enable peer-to-peer communication between instances. After the system is activated, it works in the background. The client is controlled by Session Manager. TI Applications are Java or JavaScript applications stored on a Web server. All applications can be accessed and downloaded from Application Server by the clients as needed.

TI Session Manager is the control centre for the entire collaborative system. Internally, Session Manager provides advanced peer-to-peer computing infrastructure supporting event distribution and security, messaging, and controls other collaboration tools.

TI and most of the applications are written in Java, but the TI applications can also be written in other programming languages such as $\mathrm{C}, \mathrm{C}++$, and LISP. TI provides APIs for users to develop collaborative applications. Using Tango APIs, messages can be broadcasted or sent to the participants in the same session. Tango's communication backbone ensures that messages between all applications in a session are properly routed.

\subsection{NCSA Habanero}

NCSA Habanero is a Java-based collaborative system developed at the National Center for Supercomputing Applications which can facilitate the synchronous communication over the Internet. 
NCSA Habanero consists of two parts: the Habanero environment and the Habanero framework. The Habanero environment consists of the Habanero server, the Habanero client, and Habanero applications. The Habanero framework consists of the Java APIs. The environment is built upon the framework.

The Habanero environment provides the collaborative virtual workspace. In the environment, a server hosts sessions and a set of clients interact with sessions using a variety of applications called Hablets. In Habanero system, the collaborative session is a set of participants and a set of associated tools that are involved in collaborative communication. Within a session, Habanero environment keep the state of all tools synchronized to all participants. The server manages the sessions to enable sessions to be recorded, persistent and accessed with restriction. The client provides the interface to define, list, create, join and interact with a session. It is also capable of interacting between multiple sessions.

Habanero is built on server/client architecture, but is different from a standard serverclient paradigm because it is based on the notion of replication (Jackson, 1999). Habanero works by replicating data and events in each client under the control of the server. The server stores the session states and distributes events among clients. State changes are then distributed among the different clients. When a new client joins a session, it is sent information about which applications are running in that session. Habanero also ensures that all clients see the same state changing events in the same 
order, which results in applications appearing the same to all clients. Habanero allows programmers to determine what a state-changing event is.

The Habanero server provides software mechanisms for serialization, arbitration, routing, and networking. Habanero server uses the "floor control" mechanism to arbitrate the process to decide the order in which the events are processed. Habanero allows each client to do its own routing. Habanero takes care of networking, and establishes the network connection by using TCP/IP for applications.

Habanero is implemented as a set of Java applications. Habanero applications communicate information through a Habanero client, which passes on information to the Habanero server, and the Habanero server then communicates this information with the other Habanero clients. The client starts by creating a session on a server. The client then requests a socket from the server. This socket contains all the messages about starting new tools, new people joining, and things exiting and quitting. In the same session, when an application tool is launched on a client, the application is also launched synchronously on another client in the same session.

The Habanero framework provides developers APIs, and developers can use Habanero APIs to develop Internet-distributed collaborative Java applications. According to the nature of the application and the collaborative interactions between users, some actions should only be seen by the user who is performing the action. Other actions should be 
propagated to all the users working on the same tool. A developer needs to decide what will be shared in the new collaborative application.

\subsection{GroupKit}

GroupKit is a groupware toolkit for building real-time conferencing application (Roseman and Greenberg, 1992), developed by the GroupLab of Computer Science Department at the University of Calgary. GroupKit allows geographically distributed or face-to-face meetings, and belongs to both same-time-same-place and same-timedifferent-place category.

GroupKit consists of a variety of distributed processes: registrar, session managers, and conference application, its architecture is shown in Figure 3.2. Each end-user has a replica of the session manager and the conference applications, while the registrar resides at a central server. The registrar is a continuously running process in GroupKit environment, used for bootstrapping collaboration. When an end-user starts a conference management process, it connects to the registrar process. The session management processes of the different end-users communicate information regarding the conference sessions and conference members towards the registrar. After the contact is made, communication between the different conference applications occurs directly, not using the registrar as a mediator. 


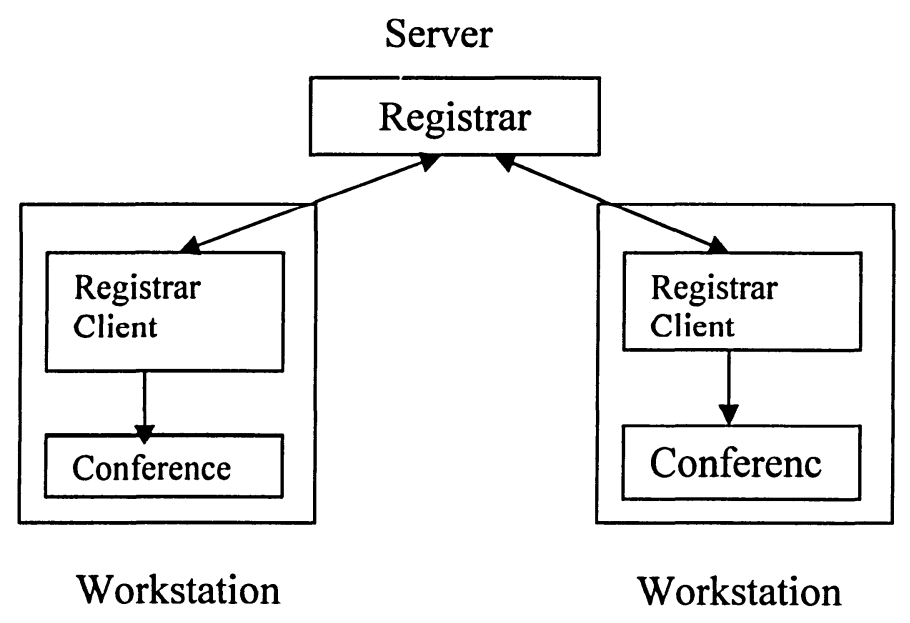

Figure 3.2 GroupKit Architecture (Roseman and Greenberg, 1992)

The Registrar is the first process created in a GroupKit session, working as the server for groupware application. Its function is to provide a central meeting point for conference management processes. For a community of conference users there is only one Registrar, and its address should be "well-known" so that other processes know how to reach it. When session manager processes are created, they connect to the Registrar. The Registrar maintains a list of all conferences and the users in each conference. It serves as an initial contact point to locate existing conference processes. The session manager controls the Registrar to add or delete conferences or users; and the Registrar then relays these changes to other session managers. The session manager provides user interface and enables users to create, delete, monitor, join, or leave conferences.

A conference application is a GroupKit groupware tool and is invoked by the user through the session manager. Conference applications work together as replicated processes, running on each participant's workstation. A process instance is a conference 
process, while the set of conference processes working together is called a conference session.

GroupKit is written in Berkeley's Tcl/Tk language and GroupKit applications can be developed using $\mathrm{Tcl} / \mathrm{Tk}$. $\mathrm{Tcl}$ is an interpreted scripting language, and $\mathrm{Tk}$ is an interface toolkit. GroupKit provides the developer with three programming abstractions to control the behavior of distributed processes, to take action on state changes, and to share relevant data which includes multicast remote procedure calls, events and environments.

\subsection{Shastra Web Based Collaborative Substrate}

The Shastra Web Based Collaborative Substrate (Shastra) is developed by the Center for Computational Visualization at the University of Texas at Austin. Shastra is an extensible, programmable environment that use the World Wide Web to facilitate large scale geographically dispersed collaboration amongst multiple participants.

Shastra consists of a collection of Shastra applications running on networked workstations and forming a collaboration environment, support cooperative work among distributed users and applications. The architecture of Shastra is shown in Figure 3.3. 


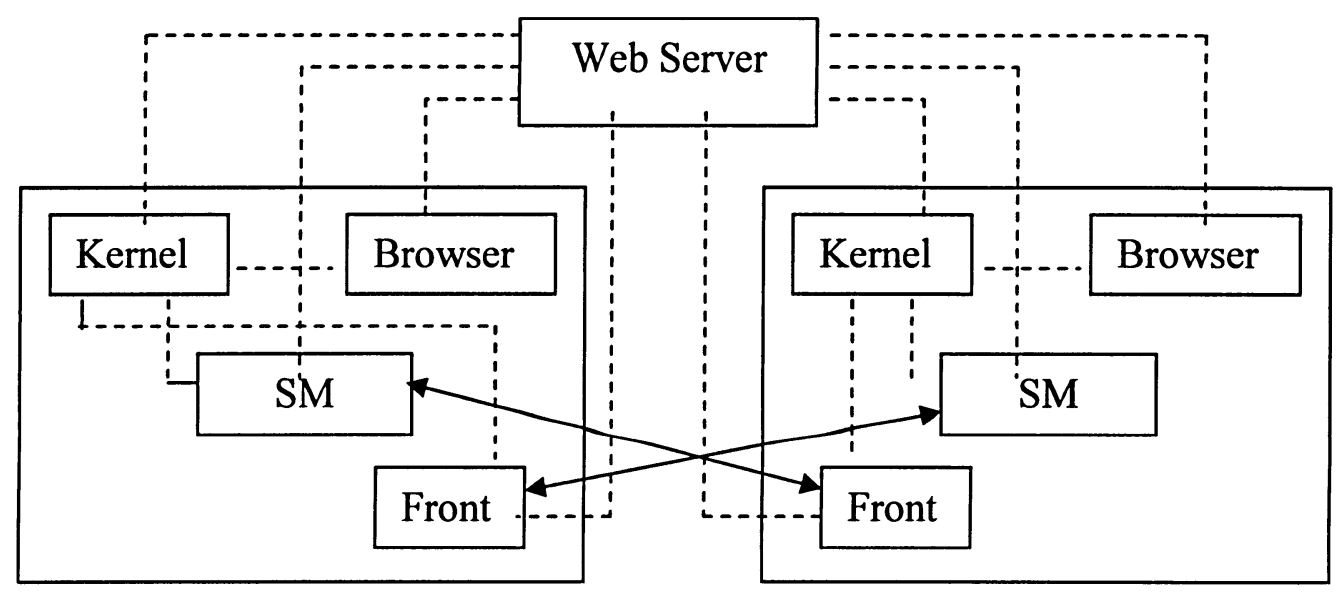

Figure 3.3 Shastra Runtime Architecture (Anupam and Bajaj, 1993)

In Shastra runtime environment, Kernel is responsible for maintenance of the distributed environment and management of network resources. Kernel instantiates service and toolkits on request from users or applications, lets users dynamically discover what services and applications are active in the environment at any time, and provides contact information about where the services and toolkits are running in the environment, enabling applications dynamically connect to each other to access toolkit functionality or to access services. The Session Manager (SM) maintains the collaboration and handles details of connection and session management. The session manager provides the broadcast facility needed for information exchange in multi-user synchronous conferencing. Thin Clients are Web browsers under the control of a session manager to provide for multi-client synchronization. Fronts are application and service processes running in the Shastra environment. Front can access the Shastra environment to instantiate tools locally or on remote sites, and to terminate previously created tools. Fronts can also connect directly to each other to exchange data in client-server settings. 
Shastra applications are written using a collection of libraries that provide efficient mechanisms for performing necessary collaborative and distributed tasks (Anupam and Bajaj, 1993). The Shastra provides a collection of Java packages that allow programmers to quickly and easily create collaborative applications that execute within the Shastra interaction environment. The packages provide collections of classes that implement various types of Ports, Servers, and Services which developers can use to implement specialized collaborative applets (Anupam and Bajaj, 1993).

\subsection{Evaluation and Summary}

In this chapter, four main academic collaborative frameworks were described and analyzed. All of them provide developer APIs or libraries that enable developers to build collaborative applications and integrate into the collaborative system.

In this research, TANGO Interactive software is only granted one year license period. After the license expiration date, TANGO Interactive Meeting Engine will not start. The license only allow two concurrent users, TANGO Interactive Meeting Engine cannot accept more concurrent users than the limit defined in the license. The offered TANGO Interactive APIs and TANGO Interactive framework are incompatible. The applications development cannot be conducted with the user API. These limitations made TANGO Interactive cannot be used as the development framework in this research.

NASC Habanero software is available (including the source code) for free with no license limitation. The developer's APIs are compatible with the framework. But Habanero was 
developed using Java Develop Kit (JDK) old versions. This version of Habanero cannot be run in the computers with Pentium 4 CPU. All source codes of APIs need to be recompiled under JDK new version and those deprecated Java classes should be modified and rebuilt. The Habanero environment should be redeveloped with the rebuilt APIs.

Though Shastra Substrate enables developers to build web-based collaborative applications, the source code of Shastra Substrate and its libraries are not available to this research. GroupKit application should be developed by Tcl/Tk language which cannot be used to develop Java application.

Table 3.1 illustrates the comparison of main characteristics and features of the four collaborative frameworks studied in this research.

Table 3.1 Comparison of Collaborative Frameworks

\begin{tabular}{|c|c|c|c|c|c|}
\hline Framework & Type of API & $\begin{array}{c}\text { Application } \\
\text { developing } \\
\text { language }\end{array}$ & $\begin{array}{c}\text { Support } \\
\text { MapObjects } \\
\text { APIs? }\end{array}$ & $\begin{array}{c}\text { Availability of } \\
\text { Framework }\end{array}$ & $\begin{array}{c}\text { Availability of } \\
\text { Source Code }\end{array}$ \\
\hline TANGO & Java & Java, C, C++ & Yes & 1 year License & No \\
\hline Habanero & Java & Java & Yes & Yes & Yes \\
\hline GroupKit & Tcl/Tk & Tcl/Tk & No & Yes & No \\
\hline Shastra & Java & Java & Yes & Yes & No \\
\hline
\end{tabular}

In this research, ESRI MapObjects- Java Edition was selected as the developing tool to develop Java-based GIS applications. The detail of ESRI MapObjects is described in Chapter 5. Among the above collaborative frameworks, Habanero is freely available, and the source codes of its APIs are also freely available, the APIs could be used to develop 
Java-based collaborative GIS applications. So Habanero is the most suitable framework among those frameworks selected to study and selected as reference framework. However, The Habanero and its APIs should be rebuilt. 


\section{Chapter 4}

\section{FRAMEWORK DESIGN OF A COLLABORATIVE}

\section{GIS}

According to the research objectives described in Chapter 1, the intent of a Collaborative GIS (CGIS) covers not only many basic features in a stand along GIS, but also some specific features. These features are intended to support alternative generation, modeling, evaluation, and cartographic display functions in a spatial domain. With CGIS, decision making (meeting) participants can collaborate on the design and construction of various spatial alternatives and share interactive mapping tools over the Internet. The concurrent use of collaborative tools can be supported by knowledge-based techniques and intelligent software agents. The evaluation of collaboratively designed alternatives can be carried out with multi-criteria evaluation techniques enhanced by voting tools. The evaluation results can be visualized on special-purpose maps.

GIS-supported participatory decision making can be categorized into four meeting arrangements based on place and time, as described in Chapter 2, which tend to constrain and/or foster human-computer-human interaction (Nyerges and Jankowski, 2001b). Place refers to the locations of participants. Both face-to-face meeting and virtual meetings are supported and referred to as same-place and different-place meetings, respectively. Time refers to synchronous communication (real-time applications such as spoken 
conversation) or asynchronous communication (where interaction takes place over an extended period). In this research, the framework design for CGIS focuses on the same time/different place arrangement, where group interactions are synchronized in real-time, resulting in a "what you see is what I see" situation.

\subsection{System Requirements}

Based on the intension of CGIS and knowledge abilities of decision participants (as they range across experts to novices in using spatial decision support tools), the following design requirements were proposed:

i. The system should offer decisional guidance to users in the form of a problem solving agenda, listing major steps used in a spatial decision making process which include (1) problem exploration, (2) criteria selection, (3) alternative evaluation, and (4) consensus negotiation.

ii. The system should not be restrictive, allowing the users to select tools and procedures in any order.

iii. The system should offer a number of decision making exploration tools and evaluation techniques.

iv. The user interface should be both process-oriented and data-oriented to allow an equally easy access to task-solving techniques, as well as maps and data visualization tools. 
v. The system should be capable of supporting space-distributed collaborative work by facilitating information exchange among group members and voting through the Internet.

\subsection{Functional Requirements}

To support collaborative spatial decision making, various decision aid methods and tools have been synthesized from a combination of literature on management decision support systems, spatial decision support systems, group support systems, and the literature on

collaborative GIS. These methods and tools could be classified at three levels of functional capabilities:

\section{Level 1. Basic information handling support:}

- Information management: storage, retrieval and organization of spatial data and information (e.g., distributed database management system support).

- Visual aids: manipulation (analysis) and expression (visualization) techniques for a specific part of a transportation problem (e.g., shared displays of charts, tables, maps, diagrams, matrix and/or other representational formats).

- Group collaboration support: techniques for idea generation, collection, and compilation; including anonymous input of ideas, pooling and display of textual ideas, and search facilities to identify possible common ideas (e.g., data and voice transmission, electronic voting, electronic white boards, computer conferencing, and large-screen displays). 


\section{Level 2. Decision analysis support:}

- Option modeling: methods of generating decision options. These include a variety of computational models from static spatial location models (e.g., suitability analysis in GIS) through optimization models (e.g., location-allocation models) to dynamic models that predict the behavior of real-world.

- Choice models: integration of individual criteria across aspects or alternative choices (e.g., multi-criteria decision models using multiple attribute and multiple alternatives for systematically weighted rankings or preferences).

- Structured group process techniques: methods for facilitating and structuring decision making (e.g., automated group techniques, electronic brainstorming, and technology of participation).

\section{Level 3. Support for group process consistency:}

- Judgment refinement/amplification techniques: quantification of heuristic judgment processes (e.g., Bayesian analysis, social judgment analysis for tracking each member judgments for feedback to the individual or group, and sensitivity/trade analysis for comparing transportation project alternatives).

- Analytical reasoning methods: performing problem specific reasoning based on a representation of the transportation decision problem (e.g., using mathematical programming or expert systems guided by automatic mediation, parliamentary procedure, or Robert's Rules of Order to identify patterns in reasoning process). 
The methods and tools described above are listed in order of most basic to most sophisticated and treated as building blocks of a system. Level 1 techniques are likely to be used the most, and therefore, appear in software packages as basic support as they satisfy a basic cognitive need for information manipulation. Whereas techniques in Level 2 are specialized enough for some groups who have a requirement for data analysis. Level 3 techniques are the most sophisticated and are rather complicated when it comes to development.

\subsection{Architecture Design of Collaborative Workspace}

The collaborative framework adopts a client/server architecture that mostly used in various collaboration platforms. The hardware architecture model of the collaborative framework is illustrated in Figure 4.1 which consists of a server and a set of clients.

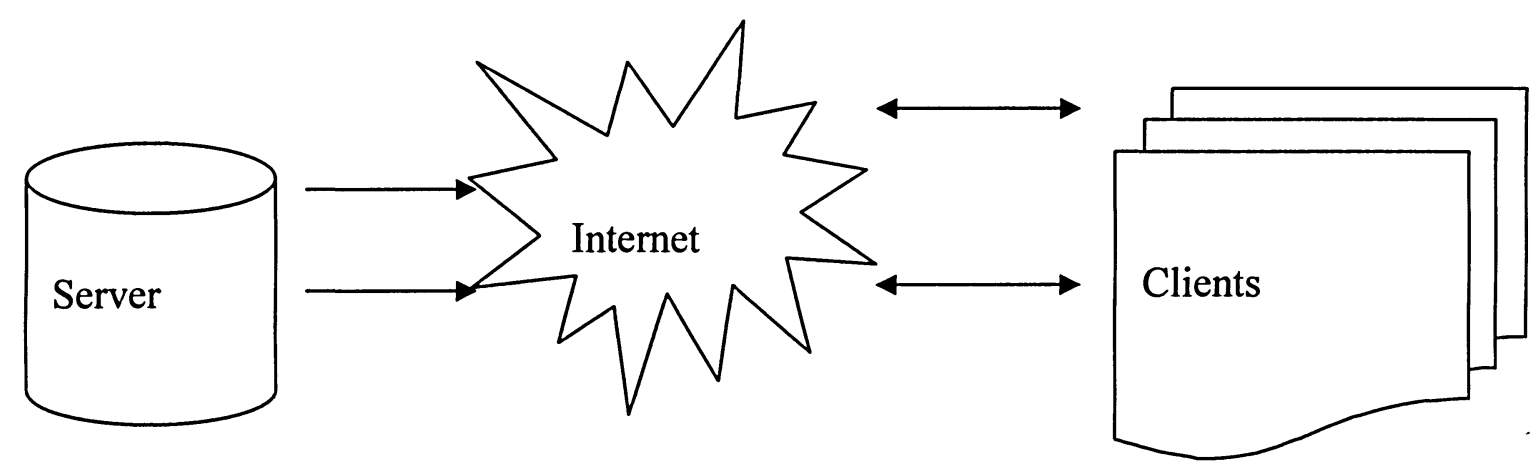

Figure 4.1 Hardware Architecture Model of Collaborative Framework

Four system elements are designed in the collaborative framework which includes:

- Session management 
- Communication among collaborating clients

- Floor control

- Synchronization

The software design architecture is presented in Figure 4.2. The details of the four elements are described in the following subsections.

Client

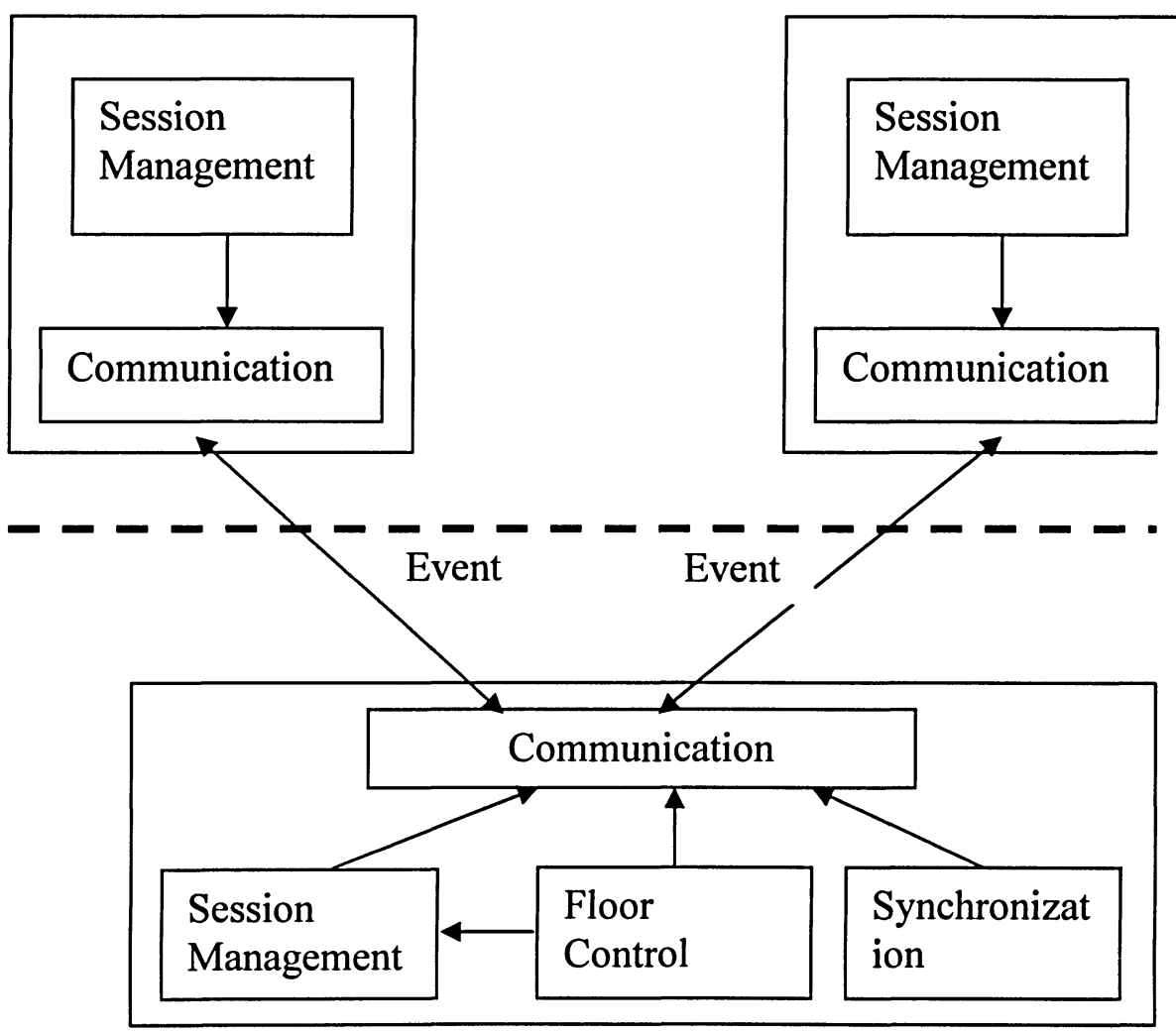

Server
The Client-side

The Internet

The Server-side

Figure 4.2 Software design architecture 


\subsubsection{Session Management}

In this research, a session is defined as a group of application instances currently working together in the collaborative mode. All applications belonging to the same session can exchange information and share behaviors. How particular application operates in a collaborative mode depends on these applications' characteristics.

Session management refers to the process of starting, stopping, joining, leaving, and browsing collaborative situations across the network. Session management should provide the following functionalities:

- Manages membership, maintains connectivity and sessions

- Makes the session creator as session master and allow session master has special privilege of controlling the session

- Displays the lists of available collaborative tools (applications)

- Controls all collaborative tools: launch and terminate of application sessions and instances on local and/or remote machines, joining and leaving existing application sessions, etc.

- Allows more than one session running simultaneously

- Displays the session status information: system users and active application sessions listings 


\subsubsection{Communication among Collaborating Clients}

Applications belonging to the same session can communicate one another. The collaborative platform should provide a basic mechanism that supports various types of communications. The communication is implemented on the basis of message passing. If a client sends a message, this message will be accepted by the server and multicasted to all other compatible applications in this particular session.

There should have two major types of messages in the collaborative platform: (a) control messages and (b) application messages. Control messages are generated by the system for communication between the server and the clients. Control messages are invisible for user applications. Application messages are the main means of communication between user applications. The structure of application messages depends on the application and is not interpreted by the communication system.

\subsubsection{Floor Control}

For collaboration to work smoothly there must be a central point of control. In this research, floor control refers to the management of interaction among participants in meetings, whenever there is a resource that must be shared among the participants, floor control issue arises. Floor control uses the metaphor of a floor, a floor denotes the temporary permission to access and manipulate resources (e.g. a shared drawing area or a video channel). Floor control uses the mechanism by which the software determines 
which user has control and how to take turns when multiple people share a limited resource such as a single cursor in a synchronous task, decides "who gets to do what when" based on the parliamentary notion of the presiding person controlling the parliamentary process. The "president" recognizes one of the participants and gives control of the deliberative floor to that participant who now has the floor. Only the participant who has the floor can send commands to the application. Floor control mechanism prevents two or more people from simultaneously clicking on different menu items and creating confusing results.

\subsubsection{Synchronization}

Synchronous collaboration in distributed interactive environment requires events from every participant to be delivered to the other participants in the same session in real time so that all participants can share a common view. A primary concern is how to hold events for synchronized execution while not hampering interactive performance.

To ensure a consistent view among participants, synchronization mechanism is used to execute the update events simultaneously in all participants in the same session. Furthermore, if the events are continuous, synchronization mechanism is also used to to ensure the order delivery of events, which results in applications appearing same to all the clients. 


\subsection{Design of Functionalities}

So far, in the related research, the methods and tools that support collaborative spatial decision making as described in Section 4.2 only have been developed as stand-alone capabilities. In our research, we designed the functionality of collaborative GIS that support collaborative decision making at three levels: collaborative mapping tools, collaborative spatial analysis tools and group decision making tools.

The collaborative GIS (CGIS) should have the basic GIS functionalities as exiting stand alone GIS (Such as ArcGIS). Generally speaking, the CGIS should be capable of performing fundamental collaborative mapping functions such as collaborative map panning map layers, zooming in and zooming out map layers, identifying vector features, searching and querying spatial features, idea generation (annotation) via mapping and text processing tools, and idea organization via editing tools.

Collaborative spatial analysis tools enable group participants propose alternatives and see the potential consequence of the proposed alternatives generated automatically by the system, or manually by any of the session participants. In a collaborative GIS environment, the result together with the original proposal should be synchronously shared with other participants. An example of spatial analysis is interactive modeling which identifies the location of a road segment for road expansion development. When one participant draws or traces a line (perhaps representing the central line of the road), 
the affected properties together with their attribute information (e.g. assessment values) are obtained and displayed on all participant's screen right away.

The key purpose of collaborative GIS is to facilitate group spatial decision making processes to achieve group consensus. The three step process of "intelligence, design, and choice" (Simon, 1976), as a general process, is essential in many decision making processes. The group decision making tool should adopt this general process. The group decision making tools should also support alternatives and multiple criteria proposing, multiple criteria evaluation of alternatives by scoring, weighting, ranking, or voting.

\subsection{SUMMARY}

This Chapter proposes the solution of framework design of the Internet-based collaborative GIS that support spatial decision making. It is proposed on the basis of system and functional analysis and requirement of collaborative spatial decision making through Internet. The framework includes collaborative workspace and functional modules that provide Internet-based collaboration, GIS module and decision making Tools. The implementation of the Collaborative GIS is described in the next Chapter. 


\section{Chapter 5}

\section{PROTOTYPE DEVELOPMENT}

\subsection{Prototype Architecture and Developing Tools}

Based on the framework design described in Chapter 4, we developed the Collaborative GIS (CGIS) prototype. The CGIS prototype contains two parts: collaborative workspace and collaborative applications.

As shown in Figure 5.1, collaborative workspace consists of server program and client program. Every client should have the same client program and collaborative applications. The server program runs in the background and does not have a graphical user interface (GUI). Its function is to link client programs during a collaborative session. Only one server program is required in a collaborative session and all participating client programs connect to that single server program. The client program has GUI which is designed to provide virtual work environments. Clients can create or join session by connecting to server program through IP address of the computer in which server program is running. In the same session, same collaborative applications in different clients can communicate collaboratively and synchronously. The working mechanism of collaborative workspace is described in Section 5.2. 


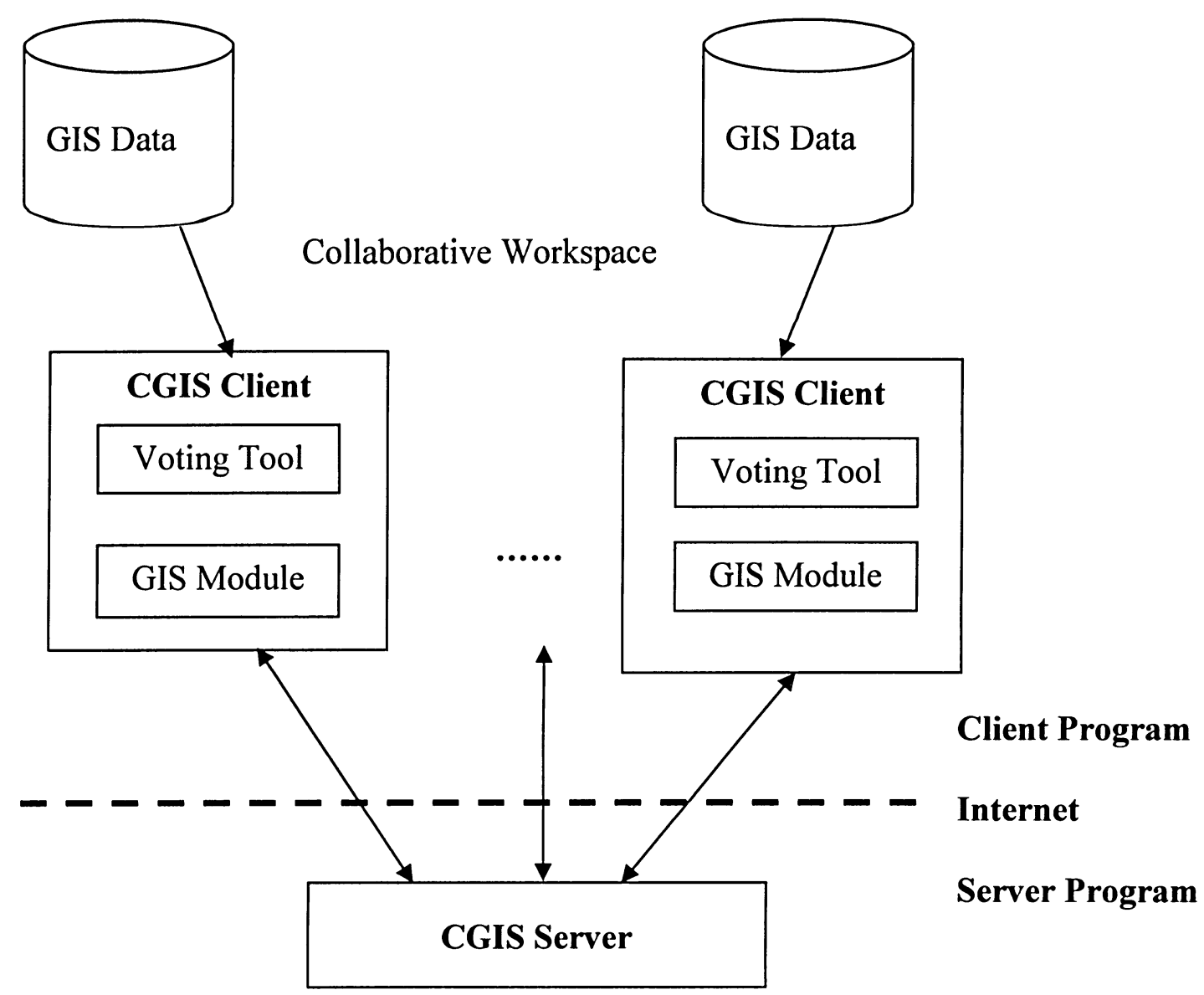

Figure 5.1 Prototype Architecture

Within the collaborative workspace, there are two collaborative applications: GIS module and Voting tool. GIS module contains basic GIS functions and spatial analysis tools, Voting tool provides the functions to perform group spatial decision making. The detail of the two collaborative applications is presented in Section 5.3.

The prototype is implemented in Java. Chapter 3 introduced NCSA Habanero framework that provides Java APIs, which contain the methods of networking facilities as well as the serialization, arbitration, and routing to be used to develop collaborative Java programs. 
In this research, a prototype of collaborative workspace is developed by taking advantage of Habanero APIs.

MapObject Java Edition is a powerful collection of pure Java components that allows developers to build cross platform, mapping and spatially enabled applications. With a robust collection of pure Java GIS and mapping components, including a suite of predefined visual JavaBeans, MapObjects Java Edition provides developers with the developing tools to develop client or server-side applications for stand-alone deployments or delivery over the Web. In this research, the Java APIs provided by MapObjects Java Edition are used to develop Java application and integrate the Java application into collaborative workspace.

\subsection{Collaborative Workspace}

According to the system requirement of collaborative workspace proposed in Chapter 4, we developed a server program and a client program. Collaborative workspace works by replicating data and events in each client under the control of an arbitrator (a software component) at a single server. Collaborative applications communicate information through the client program, which passes on information (events and data) to the server, and the server then communicates this information with the other clients. Figure 5.2 shows the process. 


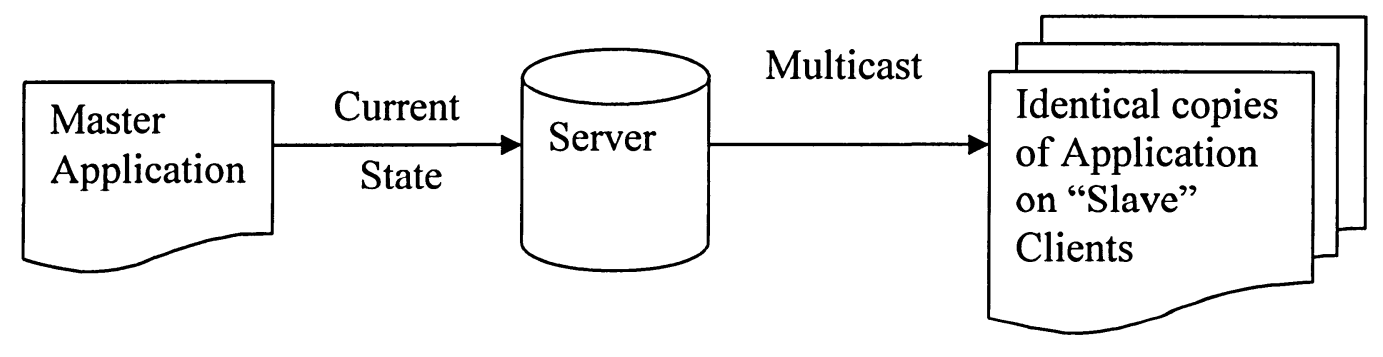

Figure 5.2 Information flow between server and client

\subsubsection{Server Program}

The server program consists of the following software components: serializers, arbitrators, and routers. These components are used to read events from and write events to a stream, controls the order in which events execute, and makes sure events get shared by everything that needs them.

Serializing is the process of copying an object. An object can be an event or data. The serializer assigns each object a numbered ticket. The tickets are used to guarantee that events happen in the same order as they are replicated on all clients.

Arbitration is the process of deciding the order in which events are processed. The server program uses a central arbitrator for every application. The arbitrator looks at every collaborative action and determines whether or not that action can be executed. The central arbitrator makes sure that all the clients see the events in the same order. Arbitrator code at the client ensures that events execute in the order prescribed by the 
tickets assigned by the serializer. Arbitrators make a TCP/IP socket connection to each client. Actions are sent to the arbitrator and then distributed to all clients.

The routers accepts events from the client and makes sure that those events go to all the appropriate clients in the same session according to those clients' IP address..

\subsubsection{Client Program}

The client program communicates with the server, which then communicates with other clients. The client program contains software components for serializing, arbitrating, routing, and networking. In essence, these duplicate the actions of the same kinds of software in the server.

The client provides the capabilities to define a new session and to participate in existing sessions, the GUI of this function is shown in Figure 5.3. In the latter case, the client provides a local version of the tools within the existing session. We defined three actions that can change the state of a session:

- Creating a session. Session is created by a participant. At the beginning, the session contains only one application instance. The participant who creates the session becomes automatically the master of this session. Other participants may then join this session.

- Joining an existing session. Joining an existing session by connecting to the session with already existing application instance. 
- Leaving a session. When a participant leaves a session, there are two scenarios: if the participant is not the master of the session, leaving the session means only removing given application instance from the session, if the participant is the master of the session, the session ends. All participants are removed from the session, the application ends, and the session is deleted.
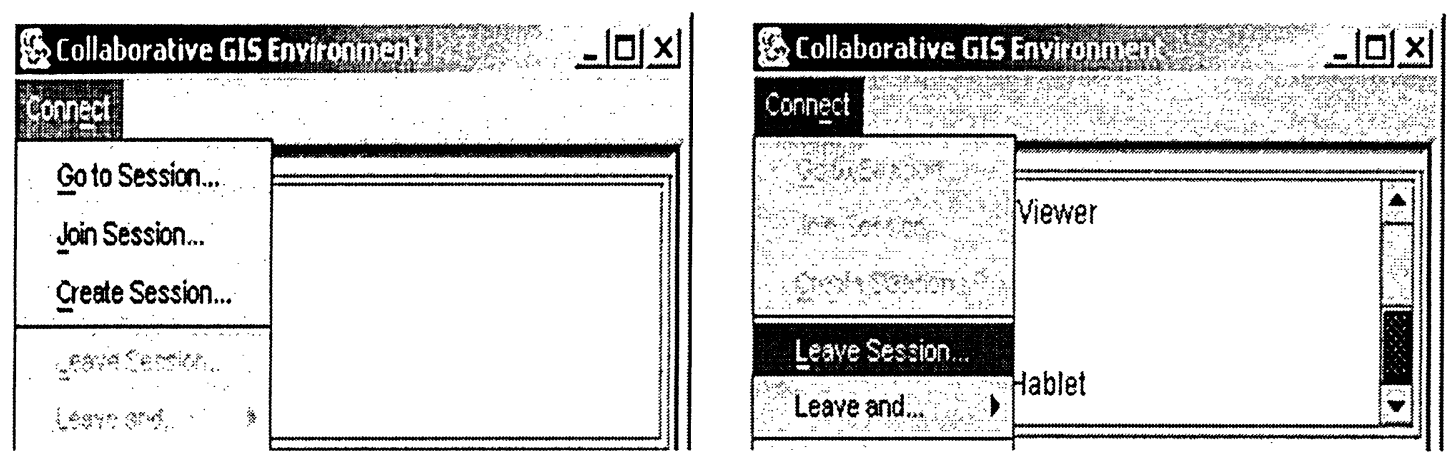

Figure 5.3 GUI of Session Status (Create, Join and Leave)

\subsubsection{Information Sharing}

The key purpose of the collaborative workspace is to share events among the applications in the same session. In our research, we took advantage of event-sharing architecture described in Section 2.2.3 to develop collaborative workspace and applications. When an event happens on a client, the task of collaborative workspace is to share that same event with all the other clients. The medium of information exchange in collaborative workspace is an action. The executable data structure can store anything from mouse clicks and key presses to images and user defined objects. The client's choices are transparently sent to the arbitrator. In collaborative application, we can create a new 
action or create an object that can be written to and restored from, a stream. The object is delivered to a specific method on all clients. Arbitrators that only process small actions often share a pipe. Arbitrators processing larger actions may request a pipe, allowing other arbitrators to continue processing smaller actions. When running collaborative application, the application can requests permission to perform a class of actions and the application sends the specific action to be shared. The action then is sent to an arbitrator and distributed to all clients. The arbitrator examines the action to determine if it is legal. Illegal actions are discarded, and legal actions forwarded to all clients. Within the client, actions are executed in the order of examination by the arbitrator.

A "button-press" example, when a button is pressed on a client running a session of collaborative application, can illustrate the above information sharing mechanism. The session has multiple listeners associated with it. Listeners listen for events and then send on the event. One of the listeners thus takes note of the button being pushed and sends the information to a listener manager. Although there are several listeners associated with each listener manager, there is a one-to-one correspondence between listener managers and sockets. The listener manager sends the event "a button has been pushed" off to the server.

At the server, there are multiple listeners as there are on the client. A listener receives the event "a button has been pushed" from the client. The server processes this event, giving it a numbered ticket and an "action_granted" message. The ticket and the "action_granted" message are sent to all other clients. A listener on the client receives the 
"ticket" and the "action_granted" and then passes them to the target, which is the correct button on the correct collaborative application.

We use a hierarchical naming scheme to ensure that events from one client are shared with the corresponding part of all other clients. The hierarchical naming scheme makes use of the frames in which objects live and the collobject to which a frame is attached. A collobject is a data type supported by Habanero API, an internal pointer.

Each session has a parent, which is the session manager. The session manager is the root of the hierarchy. The hierarchy progresses from the session manager, to the session, to the collobject, to the frame which contains the collobject, to the children of the frame, and to the object. This hierarchical naming scheme ensures, for example, that when a button is pressed on one client, the same button is pressed on all the clients.

\subsection{Collaborative Applications}

We have not attempted to reproduce all the functions of standard GIS software, but rather to provide a lightweight browser incorporating those features that are necessary in order to support interaction and discussion of spatial data, and also provide basic functions necessary to support spatial analysis and group decision making.

When developing collaborative application, there are two ways to implement the kind of synchronous collaboration. One way is to keep track of all the events that have occurred 
since an application starts up, and if a new client joins the session, to replay all those events. However, this method could take a long time to replay all the events, especially if they generate a lot of calculation. Instead, we use collaborative workspace to send the current state of the existing application to new clients that join the session. The collaborative workspace then transfers any new events to all clients. The drawback of this approach is that the application need to package up the internal state to make that state available for other clients. The advantage is that new joining clients immediately reach the same state as the other clients. In this research, we use the second way in developing collaborative GIS application.

\subsubsection{Collaborative GIS Functions}

The frame of collaborative GIS module contains three parts: toolbar, table of content and map canvas (Figure 5.4). The table of content consists of a series of legends that correspond to different layers. Depending on the type of layer, the legend also has a different presentation forms.

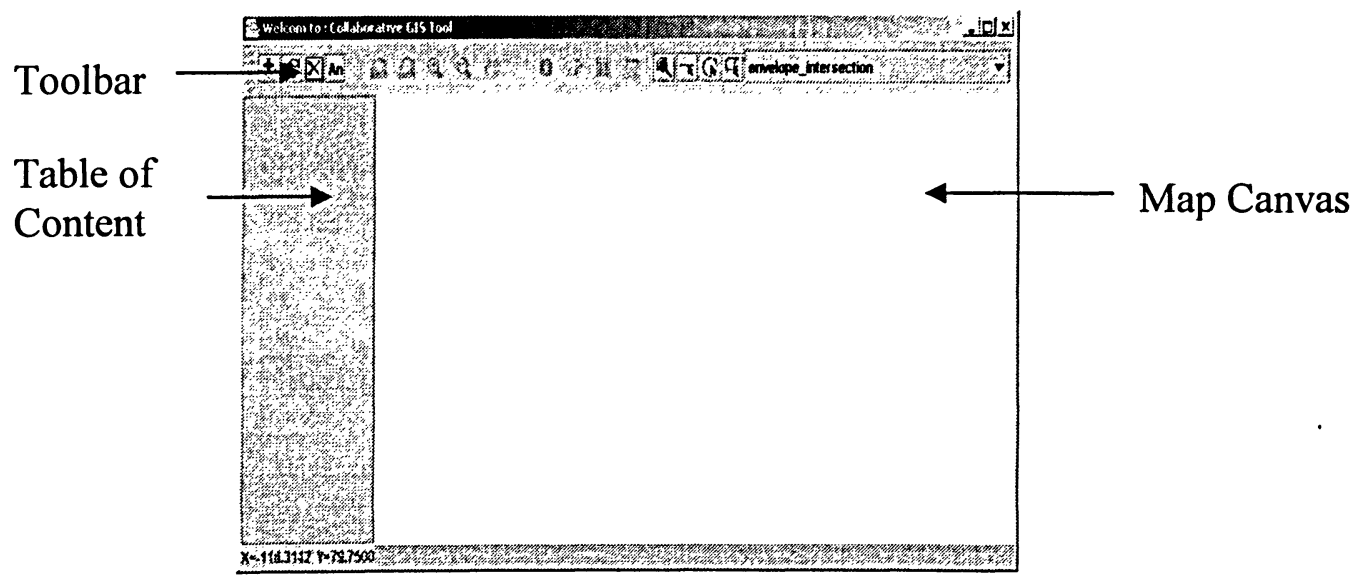

Figure 5.4 Frame of GIS Application 
Transforming coordinate methods are used to convert a pair of screen $\mathrm{x}, \mathrm{y}$ coordinates to the coordinates in map units. The MapObjects Java APIs we used to develop GIS application in this research only support world coordinate. Due to this limitation, the world coordinate is used as default coordinate system in this research.

The data formats supported by MapObjects API include shapefile, image file such as JPG and TIFF, Computer-aided design (CAD) (DGN, DXF, and DWG). Since usually the data size of image files are large and CAD file are not georefferenced, in order to save computer memory, we only allow shapefile to be identified and selected when programming the GIS application.

GIS data (shapefile) can be selected and loaded to the map canvas as a layer and displayed. The layer legend will be shown in the table of content area. When two or more participants are in the same session, one participant launches GIS application will cause the same GIS application launched in other participants' client. Any function performed by one participant will cause the same result to all participants. For example, in the same session, when one participant load spatial data into its map canvas as one map layer (Figure 5.5[a]), the same layer will be loaded into other participants' map canvas with the same coordinate system (Figure 5.5[b]). 


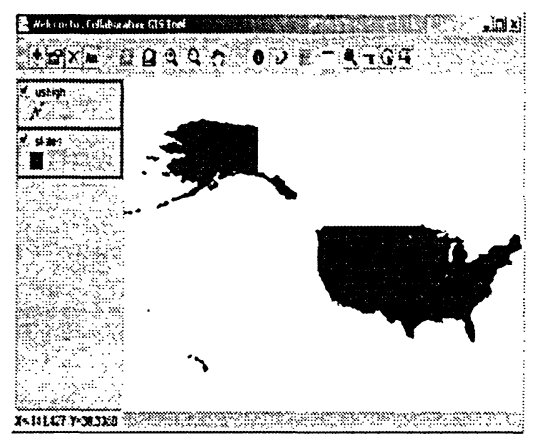

[a]

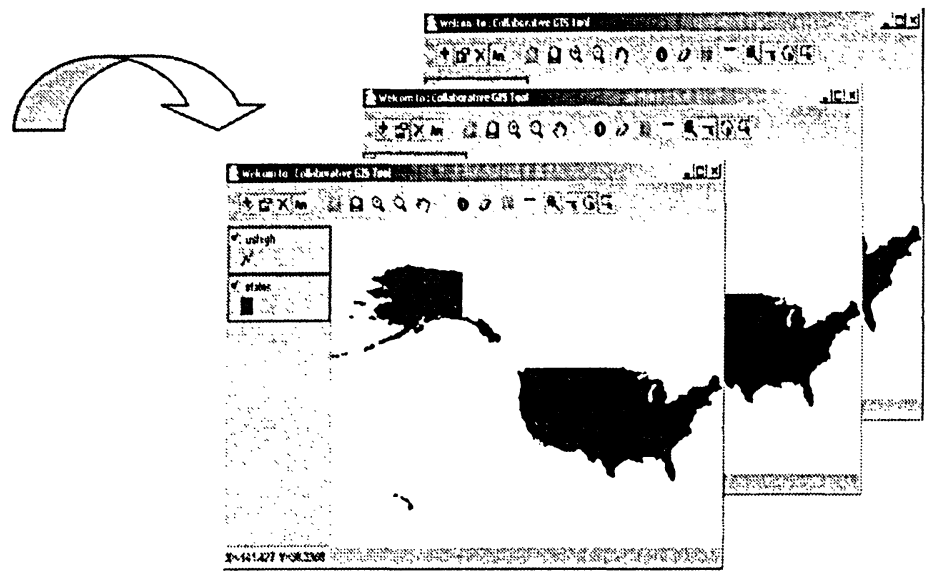

[b]

Figure 5.5 In the same session, spatial data loaded into one map canvas will also be loaded into other map canvas synchronously

The GIS module contains basic GIS tools that support spatial data browsing, retrieving and querying. These tools capture typical single-user based GIS functionalities such as loading and removing spatial data (shapefile layer), showing coordinate information, panning, zooming in and zooming out map layers, annotating, viewing the active layer's attribute table, viewing and modifying the active layer' property, identifying, searching, selecting, buffering and querying spatial features. Figure 5.6 shows the screen shot of the collaborative GIS module. 


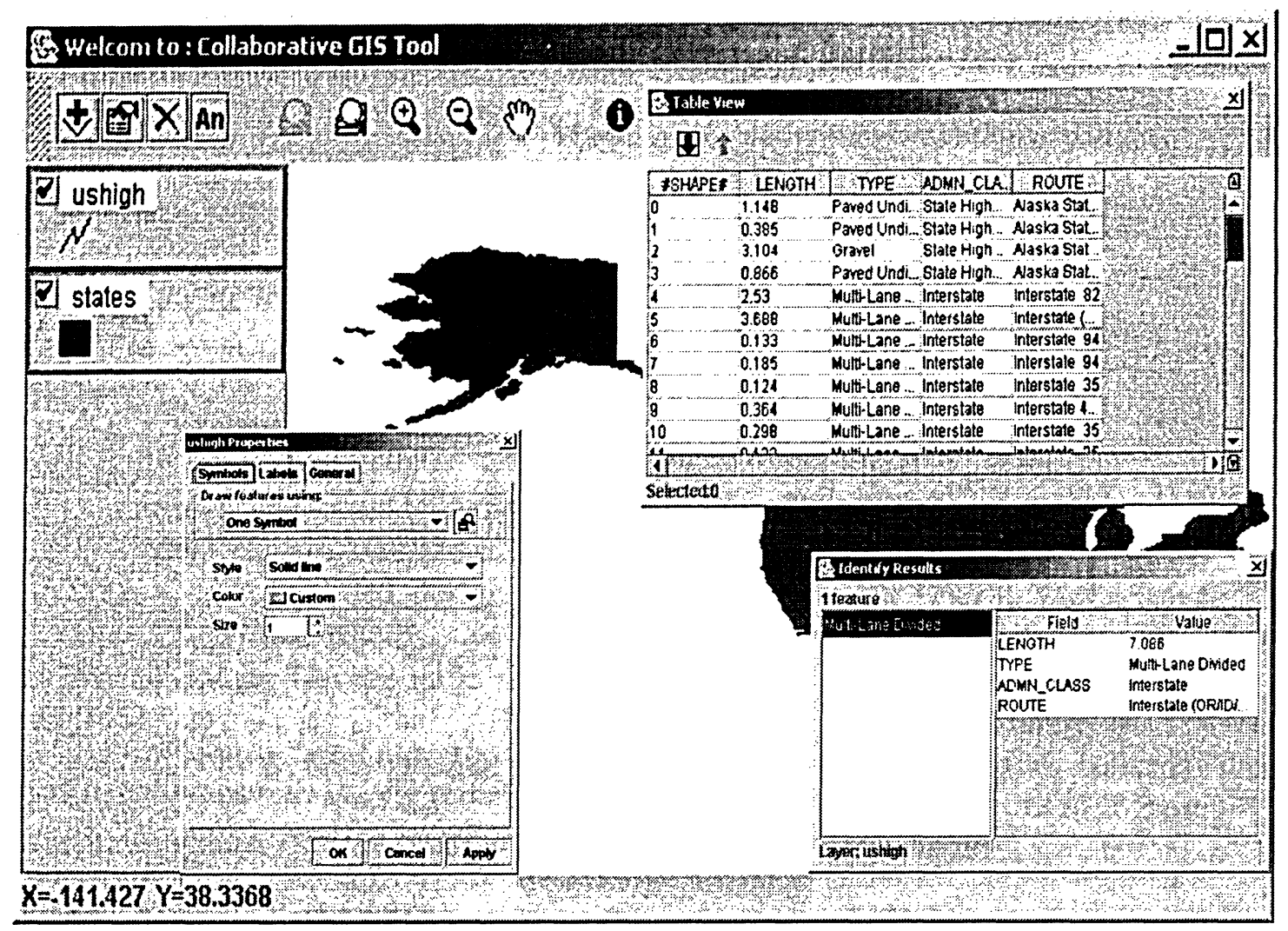

Figure 5.6 Screen shot of collaborative GIS module

\subsubsection{Spatial Analysis Tools}

We developed two types of spatial analysis tools: the first type enables spatial operations to be performed on the polyline and polygon drawn on-the-fly; the second type enables spatial analysis to be performed by selecting spatial features. These tools can perform spatial analysis such as intersection, union and difference.

An important spatial analysis tool is "interactive modeling". With which, one participant could draw or trace a polyline on the map and set up the buffer distance to this polyline. To every layer of the map, all the features covered or within the buffer zone will be 
selected, and the selected features of the active layer can be collaboratively viewed by selecting its attribute table. Figure 5.7 shows a map with two layers: US highway and US counties. When drawing a polyline with $10 \mathrm{~km}$ buffer, the selected features in the two layers are highlighted and their attribute tables are displayed.
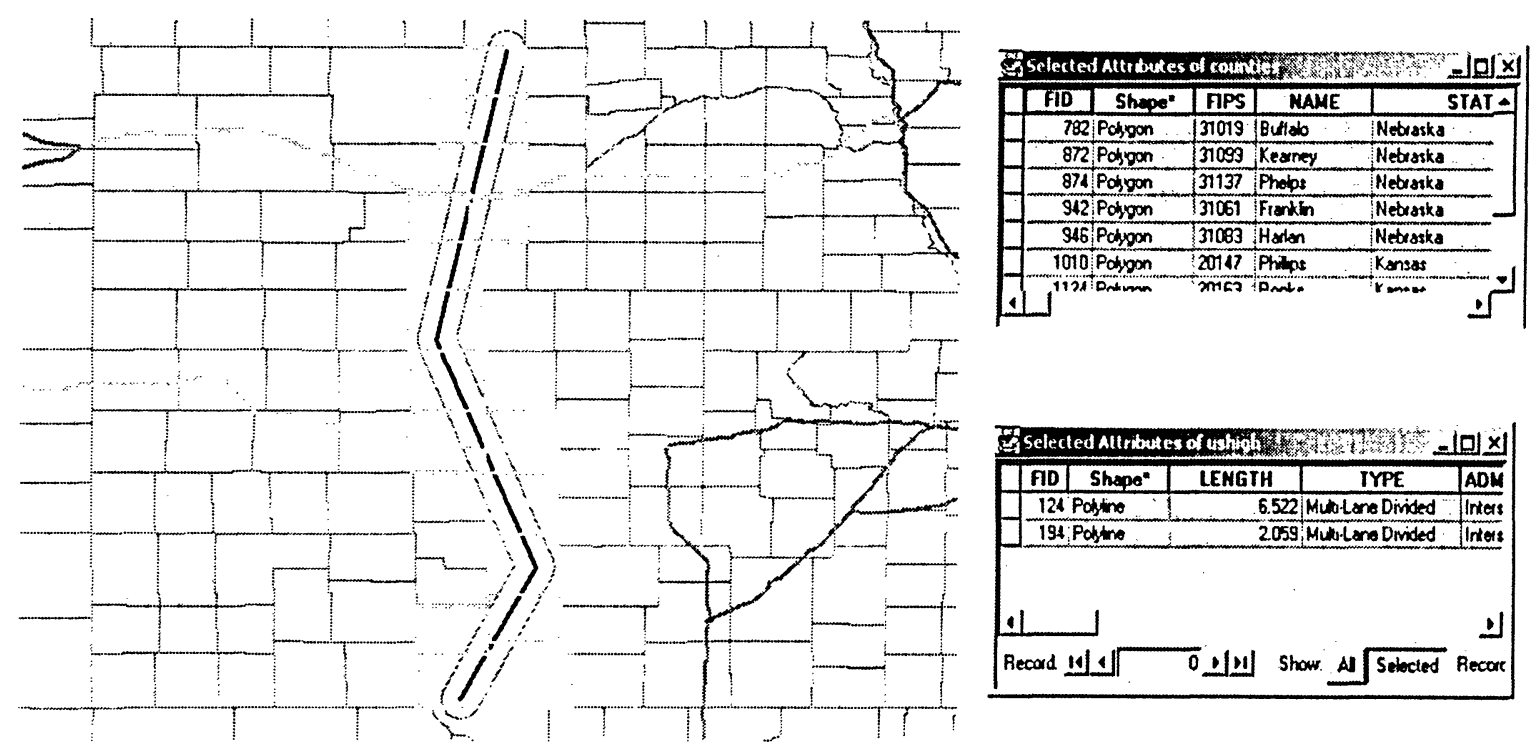

Figure 5.7 Interactive Modelling

\subsubsection{Voting Tool}

The Voting tool offers decision making guidance to group participants in the form of a problem solving agenda, it has the following capabilities:

(1) Alternative and criteria selection: participants can input their alternatives and criteria, all the alternatives and criteria will be listed;

(2) Alternative evaluation: criterion priorities are established by assigning preference scores. Scores express how important every criterion is in relation to other criteria. 
Every alternative will be evaluated by weighing all the criteria and the evaluation score is the sum of every criteria's score.

(3) Consensus voting: after all the participants finish the above steps, the voting result will be displayed to every participant. The voting result lists the alternatives, their finial score and rank.

Figure 5.8 shows the user interface of decision making process of the Voting tool.
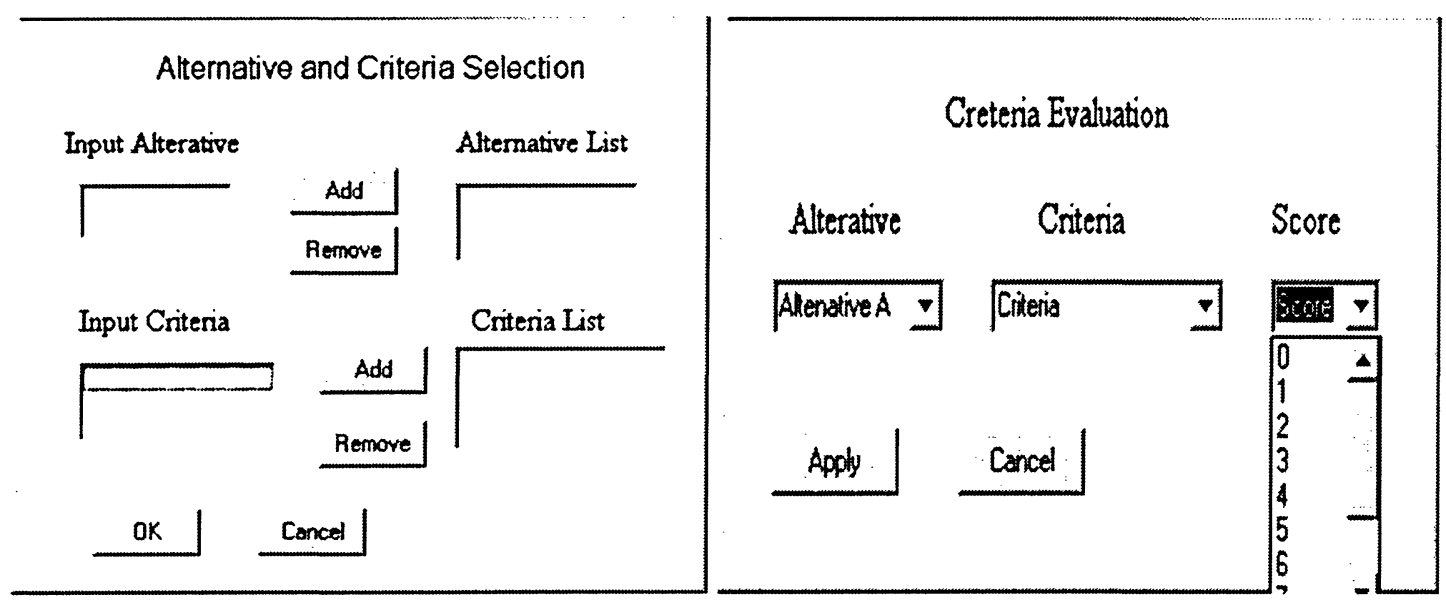

Figure 5.8 Voting Tool

\subsection{SUMMARY}

This Chapters describes the implementation of the Collaborative GIS research prototype proposed in Chapter 4. The prototype is developed with Java, ESRI MapObjects and Habanero APIs. The collaborative workspace contains server program and client program, the collaborative functions are developed at three levels: collaborative mapping tools, collaborative spatial analysis tools and group decision making tool. 


\section{Chapter 6}

\section{CGIS APPLICATION IN CORRIDOR PLANNING}

Transportation corridor planning exemplifies transportation decision making that is collaborative in nature and geographically based. Such decision making is part of a broad social trend toward shared and participatory discussions about public investment.

The purpose of this section is to examine and report on how CGIS could be used to support group-oriented decision making in transportation corridor planning.

\subsection{An Overview of Transportation Corridor Planning Process}

A corridor is a broad geographic area, defined by logical, existing and forecasted travel patterns served by various modal transportation systems that provide important connections within and between regions of the state for people, goods, and services. Travel within the corridor may include vehicular, rail, transit, water, air, or nonmotorized.

Corridor planning is a process that is collaborative with local governments and includes extensive public participation opportunities. A corridor may be divided into logical, manageable smaller areas for the purpose of corridor planning. 
Corridor planning can prioritize which transportation projects need to proceed to the programming and development stage, and to explore economical alternatives to highway construction. The purpose of corridor planning is to comprehensively address future transportation needs, and to recommend a package of improvements and management strategies for the transportation system within a corridor.

The planning process has been organized to accommodate multiple (and possibly conflicting) constituencies and needs. It accomplishes this by making all corridor planning participants go through a process that leads to:

- Understanding transportation needs

- Evaluating a wide range of solutions

- Assuring knowledge-based selection of improvements

- Balancing the needs of multiple constituencies

Referenced from the Idaho corridor planning guidebook (Idaho Corridor Planning Guidebook, 1998), the main activities in planning process including:

- Develop a corridor work plan and public participation plan.

- Research existing conditions of the transportation system.

- Analyze the projected future (20-year) travel demand and performance in the corridor.

- Establish purpose and need, and the relative importance of corridor needs through project goals.

- Generate alternatives to meet the corridor goals. 
- Identify feasible alternatives by first evaluating all alternatives.

- Use comparative analysis to further evaluate alternatives and generate a preferred list.

\subsection{A Task Model of Transportation Corridor Planning Decision Making}

Preparation of a transportation corridor planning is a rather complex group activity. The activity can involve planners and analysts come from different background and organizations, these participants have a vested interest in the outcome of the decision making process.

Although laws and transportation policies set out categories for types of projects in a nonspatial manner, the projects eventually manifest into location-based concerns. Consequently, the decision problem becomes one of site selection, based on criteria evaluation. Nyerges et al. (1997) described criteria from policy and technical aspects which could be summarized into six policy criteria (Table 6.1) and ten technical criteria (Table 6.2). The criteria cover a variety of interests and perspective, and location is only one of many criteria. However, a location-based perspective provides a natural means of evaluating the impacts that projects have on surrounding areas, and the influences that surrounding areas have on improvements. 
Table 6.1 Six Policy Criteria

1. improve mobility of urban centres or along corridors connecting centres

2. support adopted regional economic strategies

3. improve system performance, efficiencies, and effectiveness

4. reduce reliance on single occupant vehicles

5. improve existing or provide new access to ports, airports or centers

6. improve air quality.

Table 6.2 Ten Technical Criteria

\begin{tabular}{|l|}
\hline 1. maintenance and preservation \\
\hline 2. traffic congestion \\
\hline 3. mobility and connectivity \\
\hline 4. safety and security \\
\hline 5. efficiency and reliability \\
\hline 6. accessibility \\
\hline 7. economic benefit \\
\hline 8. air quality/energy savings \\
\hline 9. other environmental benefits \\
\hline 10.cost assessment. \\
\hline
\end{tabular}


Based on the description of the corridor planning processes from the previous section and referenced from the group-based spatial decision making task model developed by Nyerges et al. (1997) and Nyerges and Jankowski, (2001b), we developed a task model for summarizing the corridor planning process (Figure 6.1). This task model summarizes the process-based needs for information technology support, and allows us to establish more easily a requirement specification for collaborative GIS to meet those needs. The task model depicts transportation corridor planning as a process of social and political interaction among participants in a group. An advantage of setting up a task model for a decision making process is that it is possible to provide details for the process in an incremental manner and develop information technologies that facilitate the workings of such a process in an evolutionary manner.

Input

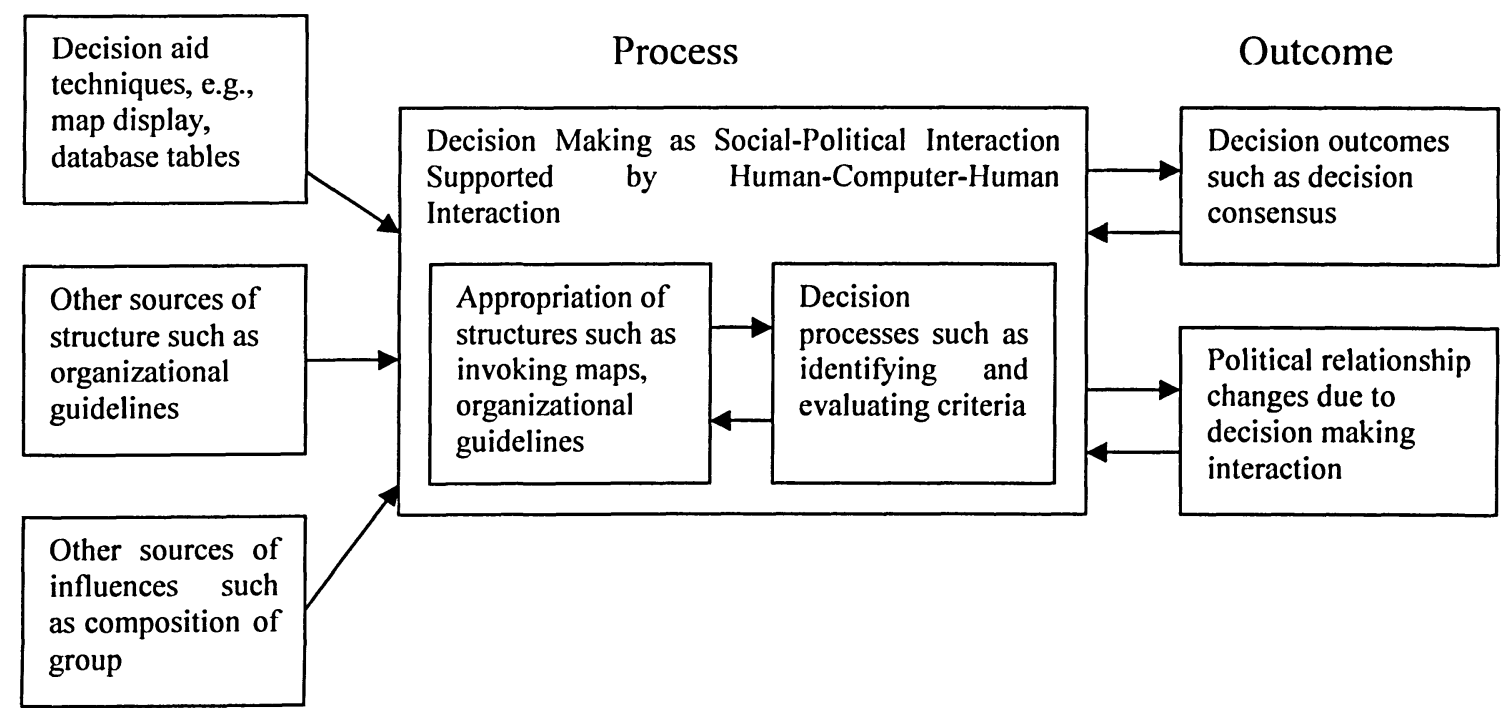

Figure 6.1 A task model for collaborative GIS technology in support of corridor planning (After Nyerges and Jankowski, 2001b) 
As with any decision process, it is important to avoid oversimplification in transportation decision making. However, it is useful to abstract information about the process and simplify it in order to be clear about this topic. The normative process for transportation corridor planning can be described in terms of three general steps: an initial problem screening where criteria are identified; the use of criteria during evaluation and the selection of alternatives through socio-politically charged conversations. This three steps process identified here is much like that presented by Simon (1976) as a general process of "intelligence, design, and choice", respectively, with the updated recognition that each of the steps is influenced by multiple values when placed in a broader stakeholder group setting (Simon and Associates, 1992).

\subsection{Empirical Case Study}

The case study area is related to serious traffic problem in Toronto central area. The assumption is that a new highway is under planning to release the traffic problem. A group of planners who come from different organizations and have different backgrounds perform the planning task. Those decision making participants are in different locations. Firstly, they launch the CGIS client program separately from their computers and connect to the CGIS server program through the server's IP address on which the server program is running. Then one of them creates a new session and rest of them joins this session. In this session, the collaborative GIS application and Voting Tool can be launched by any participant from his collaborative workspace. The same applications will be also launched on other participants' collaborative workspace. Next, they load different layers 
focus on study area into the map canvas separately from their own aspect and interest. It is further assumed there are four different layers loaded by different participants including Road layer, Water layer, Vegetation layer and Land Use layer. Figure 6.2 shows the layers in study area. The redundant layers can be removed from the map canvas by the session master.

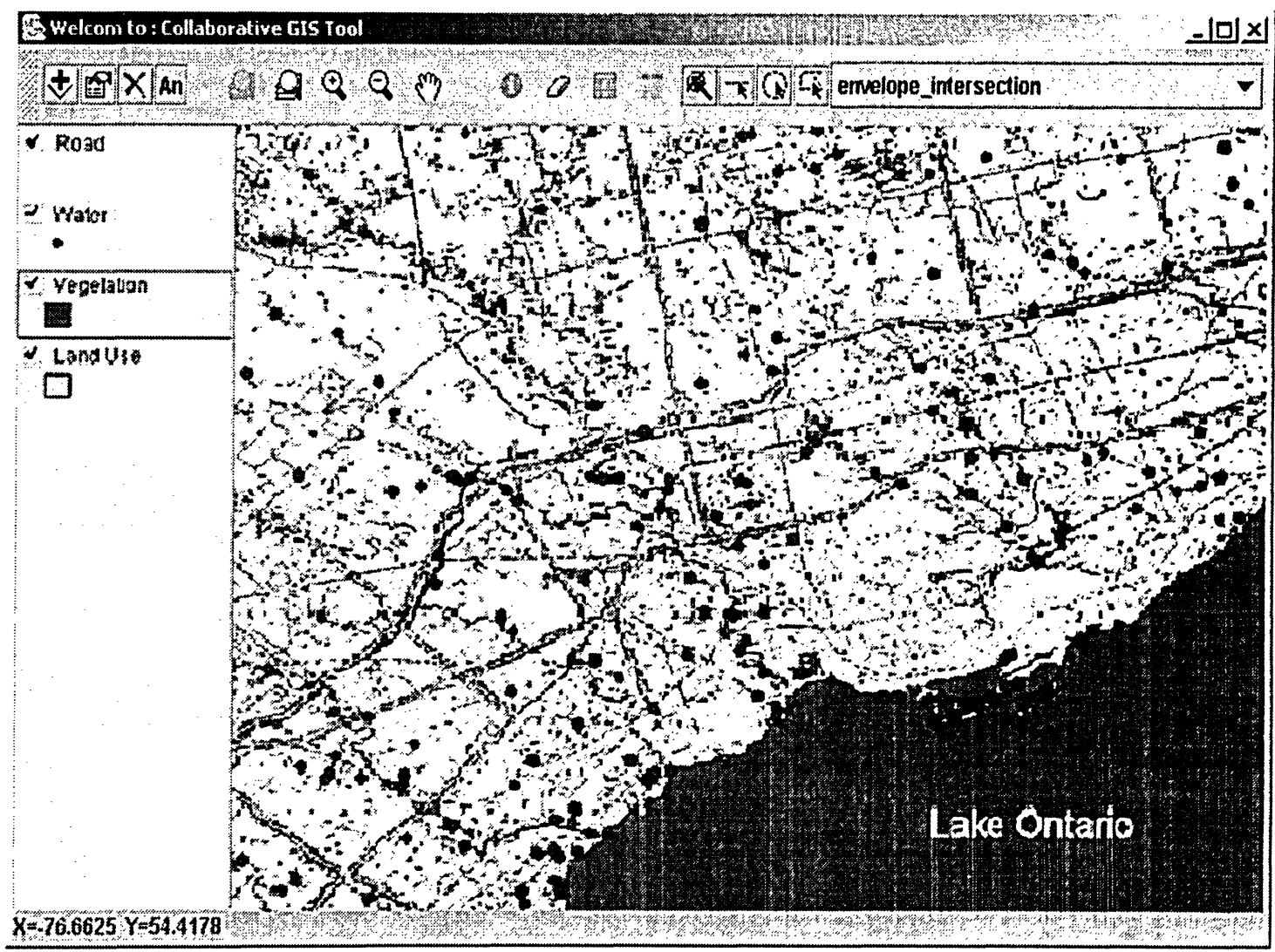

Figure 6.2 Study Area (contain 4 layers: Roads, Water, Vegetation and Land Use)

The participants can explore the maps and their attributes data, and then separately use Interactive Modeling tool to draw a polyline with buffer distance 50 meters as a transportation corridor, and annotate the alternatives. All the alternatives will be displayed in every participant's map canvas (Figure 6.3[a]). Participants can also check 
the features in the map layers that are covered by the buffer zone (selected features means they are affected by the corridors) (Figure 6.3[b]).

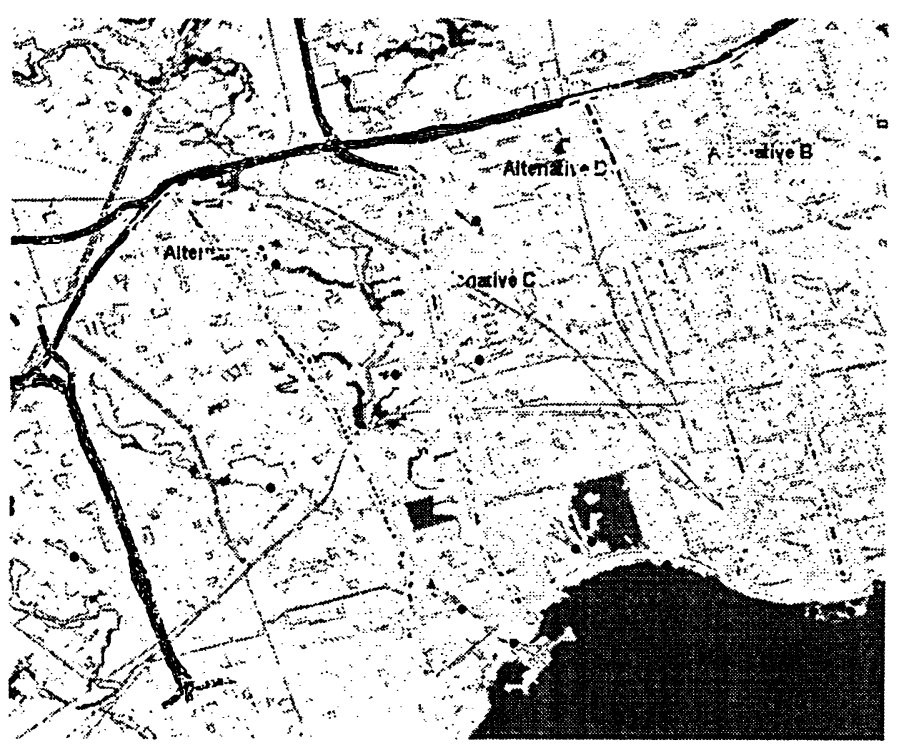

[a]

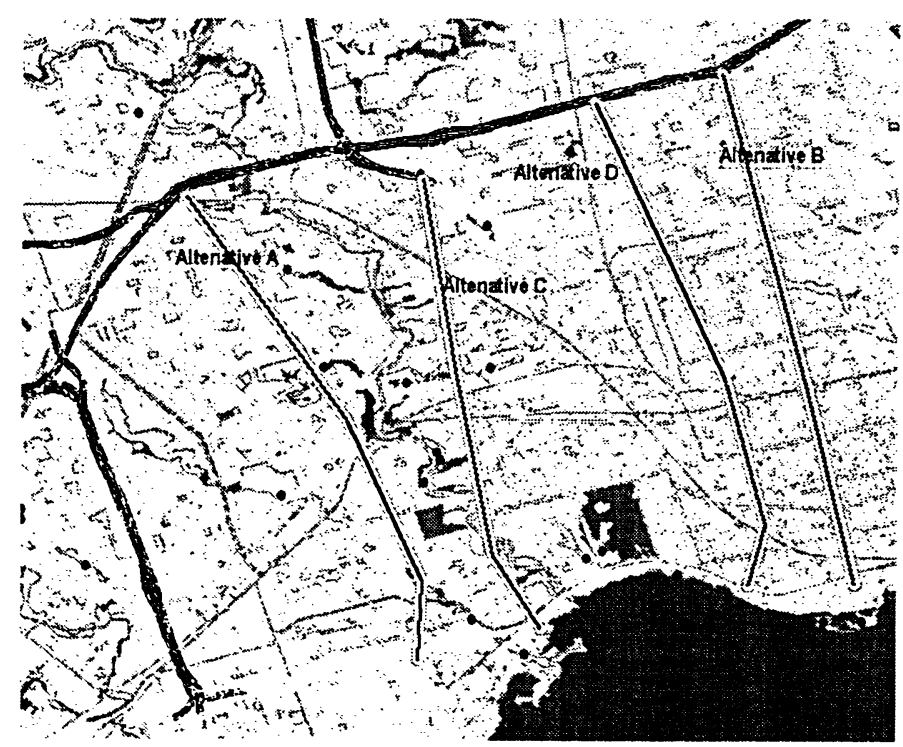

[b]

Figure 6.3 Four Alternatives and the affected features 
After proposing the alternatives, the participants then use Voting tool to get the consensus. The decision making procedure follows the following steps: firstly, every participant input his/her alternative's name and the preferred criteria (Figure 6.4[a]). After all the participants finish this step, they are provided with a list of participating computers' IP addresses, all the alternatives and all the criteria (Figure 6.4[b]). Participants then measure those alternatives against the list of criteria by assigning the score from 0 to 10 (Figure $6.4[\mathrm{c}]$ ). The more important the criteria, the higher the score assigned. A matrix $S_{[i, j]}$ is used to store the score. $S_{[i, j]}$ means to alternative $j$, the sum of the score assigned by participant $i$. When all the participants finish evaluating all the alternatives, the voting result that lists the final score and rank of those alternatives is provided (Figure 6.4[d]). To every alternative, the value of the final score is the average of the sum assigned by every participant. 


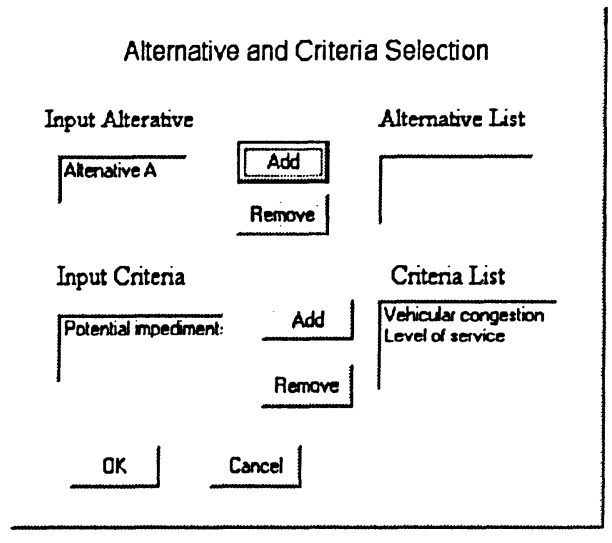

[a]

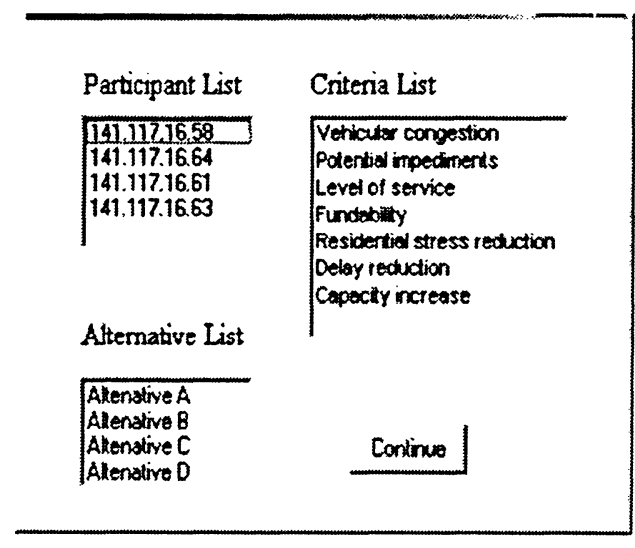

[b]

Voting Result

Alternative Scroe Rank

$\begin{array}{lll}\text { Altenative A } & 53 & 2\end{array}$

Altenative B $\quad 49$

$\begin{array}{lll}\text { Altenative } \mathrm{C} & 58 & 1\end{array}$

Altenative D $\quad 45$

[c]

[d]

Figure 6.4 Voting Process

Because of the research limitations described in Chapter 1, the performance test of the CGIS prototype only was performed under lab condition and the participants are graduate students of Geomatics program who play the role of decision makers come from different organizations. 


\subsection{Summary}

This case study indicates that there are considerable potential benefits associated with the use of a collaborative GIS in spatial decision making. Using CGIS does not resolve conflict in itself, rather it helps discover some possible areas in which some type of consensus might be achieved by helping to "visualize common ground" among priorities. Political decisions that favour some groups over others are still likely to be part of collaborative transportation decision making. Undoubtedly the backgrounds and agendas of participants involved in the decision studies will influence the result. Better understanding of the behavioural and social issues in the context of information technology use will foster better task model designs. 


\section{Chapter 7}

\section{CONCLUSIONS AND RECOMMENDATIONS}

This thesis describes a new approach of designing and developing an Internet-based synchronous GIS for supporting collaborative spatial decision making. The focus is on solving how GIS and groupware can be integrated to support collaborative activities and real-time decision making. This thesis has resulted in a research prototype called CGIS, developed based on the designed framework.

In this chapter, I briefly summarize the work described in the previous chapters, followed by discussion on the results obtained. Since our research is only based on limited studies and methods employed, we discuss the future work about the development of collaborative GIS which could improve the research outcomes and implications of further usage of collaborative GIS in a wider area.

\subsection{Conclusions}

The goal of this research was to develop an Internet-based collaborative GIS to support group spatial decision making. The related works were reviewed in Chapter 2. A number of specific issues were identified and are examined in the process of achieving this goal. These issues include: 


\section{Selection of Collaborative Framework for Developing a Collaborative Workspace}

To support synchronous and distributed spatial decision activities, we need to develop an Internet-based collaborative GIS. From the prospects of time and cost, it was not feasible to develop a new collaborative system. The collaborative workspace should be developed on the freely available groupware. To accomplish this objective, four existing free collaborative frameworks were investigated and evaluated in Chapter 3, and NCSA Habanero was selected not only for its available Java APIs that could be used to develop collaborative program, but also its possible compatibilities with the Java-based GIS application development tool - MapObjects Java Edition.

\section{Design the Framework of Collaborative GIS}

The design guideline of the collaborative GIS was proposed on the basis of the theory of GIS-supported collaborative decision making that was introduced in Chapter 2. The framework of CGIS contains collaborative workspace and collaborative applications. The collaborative workspace was designed as the platform on which events and actions could be performed collaboratively. The collaborative applications are designed to provide collaborative tools to support GIS data exploration and manipulation, spatial analysis and spatial decision making. 


\section{Prototype Development of Collaborative GIS}

The accomplishment of this objective was addressed in Chapter 5. The collaborative workspace adopted client/server architecture due to the distribution character of collaborative GIS. The collaborative applications consisted of GIS module and Voting tool which provide the functions required in the framework design. The most difficult part in developing collaborative GIS was the incompatibility between Habanero APIs and MapObjects APIs. A lot of work spent on re-programming and recompiling Habanero APIs. Developing a collaborative system to support spatial analysis and decision in a distributed work environment is a huge task requiring extensive research, which cannot be accomplished within one thesis. This is mainly because of the design and implementation complexity of CSCW and groupware tools in real-world applications.

\section{Prototype Test with Case Study of Corridor Planning}

The case study, as discussed in Chapter 6, was in an attempt to verify whether or not the collaborative GIS can facilitate collaborative spatial decision making. This case study used a task model to explicitly incorporate social and organizational design constraints (opportunities) that inform the technological design considerations. The testing results are positive and beneficial in the application of corridor planning:

The research has a strong implication in group-based GIS participation, which has an intention of providing spatially enabled participatory tools for supporting group 
participation in any decision making efforts affecting public interests. These tools provide such capabilities as rating participation, voting on public issues, sharing spatial views of different arguments, and communicating with decision makers and other participants.

The developed collaborative prototype facilitates the use of emerging technologies such as groupware and GIS application development tool to improve the efficiency of the collaborative spatial decision making by ensuring information concurrency, accessibility and availability.

The computer-based coordination and collaboration mechanisms, or CSCW technologies, was initially created to support information sharing among group of people for better and faster decision making. In this prospect, decision making requires not only capabilities of sharing necessary information but also capabilities of supporting good decision making processes ( $\mathrm{Li}, 2003)$. In many group activities, decision makers may be located at various geographical locations. The need for distributing required information and controlling decision making processes across wide areas becomes significant. Especially when the decision making efforts involve spatial representations of scenarios to be selected, capabilities of collaboratively viewing these representations and making comments on top of them offer more facilitations than those offered by typical means such as telephone meetings and electronic meeting systems.

In summary, this research integrated GIS, Internet and groupware in one workable prototype, and applied them to spatial decision making. The research results indicate that 
such prototype enabled collaborative spatial decision making, provided viable participatory tools to support group participation GIS through enhanced real-time communication. The research results also indicated developing collaborative workspace is a hard task. The key issue of developing a collaborative GIS is to develop a new or use a freely available collaborative framework with which collaborative applications could be integrated smoothly.

\subsection{Recommendations for Future Research}

Several issues related to the research prototype to provide better solutions to support collaborative spatial decision making need further investigations, developments and evaluations. These issues are discussed as follows:

\section{(1) Spatial Data Management.}

Spatial data management is one of the three core geographic information technologies because of the large amounts of spatial data being processed. Group-based GIS has strong requirement for accessing to the shared data and having a group review of the basic data categories plus attributes, hence criteria that can be used to frame a decision problem. Although all GISs have basic data management capabilities, few GIS packages have distributed data management capabilities built into the spatial data manager component of the system. Oracle and MS SQL server are example database

management systems that support attribute data retrieval and storage across local computer networks and Internet, among which Oracle Spatial can store and manage 
spatial data directly. Among numerous languages that support database design, the Unified Modeling Language (UML) has been used by ESRI (vendor of MapObjects) to specify system and module design, and promote geospatial database design. These database design and management technologies should be considered in the future research to achieve more effective data sharing during group visualization and analysis processes.

\section{(2) Choice Model Techniques.}

Choice models provide assistance in comparing numerous alternatives against set of criteria to select the best alternatives. Integration of multi-criteria decision (MCD) models with GIS has been an active area of research in the past few years (Jankowski, 1997), and is now becoming a development activity. The integration of multi-criteria decision models into CGIS should be the development activity in the further research. For example, the MCD model proposed by Jankowski (1997) could be considered in the future development. This is a weighted summation technique which is based on a linear combination of criterion scores and weights.

\section{(3) Structured Group Process}

In this research, CGIS has been developed to help facilitate group interaction, but did not make explicit use of structured group process techniques. Structured group process techniques, together with the techniques for collaborative communication support, can be used to organize meetings for structured creativity. Such techniques are used to encourage group process direction, often useful at the beginning of group 
processes where information creativity and planning tasks are more common than at the end of group processes. More research must be done to enhance the spatial decision process in collaborative GIS.

\section{(4) Application in Real World}

The overall performance of the collaborative GIS should be tested and analyzed in real-world environments to obtain better understanding on improved efficiency and effectiveness in collaborative decision making activities. In real-time environment, the participants may use the collaborative GIS with different Internet access levels (such as cable, dial-up, wireless), because the different Internet access speed could cause the delay or other problems in collaborative session. Overcome such factors that may effect the synchronous collaboration should be considered in the future research. 


\section{REFERENCES}

A. Chabert, E. Grossman, L. Jackson, and S. Pietrovicz 1997. NCSA Habanero: Synchronous collaborative framework and environment. In Conference Supplement of the Fifth European Conference on Computer-Supported Cooperative Work, pages 7-18, Lancaster, UK.

A. J. Dix and R. Beale 1996. Information requirements of distributed workers. In Remote cooperation: CSCW issues for mobile and tele-workers, Eds. A. J. Dix and R. Beale. Springer Verlag. pp. 113-143.

Anúpam V. and Bajaj C. 1993, SHASTRA - An Architecture for Development of Collaborative Applications, Proceedings of the Second IEEE Workshop on Enabling Technologies: Infrastructures of Collaborative Enterprises, Morgantown, West Virginia, IEEE Computer Society Press, p. 155-166.

Arias, E. G. and Fischer, G. 2000, Boundary objects: Their role in articulating the task at hand and making information Relevant to It. International ICSC Symposium on Interactive and Collaborative Computing (ICC'2000), Wetaskiwin, Canada, December 2000, pp. 567-574.

Armstrong, M. P., Densham, P. J. and Rushton, G. 1986. Architecture for a microcomputer based spatial decision support system, Second International Symposium on Spatial Data Handling, Vol. 120-131 Int. Geogr. Union.

Armstrong, M.P. and P.J. Densham, 1990. Database organization alternatives for spatial decision support systems, International Journal of Geographical Information Systems, Vol 3(1), pp57-65.

Armstrong, M. P. and Densham, P. J. 1995. Cartographic support for collaborative spatial decision making.Auto Carto 12, ACSM/ASPRS Technical Papers, Vol. 4, Charlotte, NC, Feb. 1995, pp. 49-58.

Armstrong, M. P. 1994. Requirements for the development of GIS-based group decisionsupport systems. Journalof the American Society for Information Science, 45(9), 669677.

Armstrong, M. P. 1993. Perspectives on the development of group decision support systems for locational problem-solving. Geographical Systems, 1(1), 69-81.

Baecker R M 1993 a. Readings in Groupware and Computer Supported Cooperative Work: Assisting Human-Human Collaboration. San Fransisco, Morgan Kaufman. 
Baecker, R.M. 1993 b. Part I: Introduction." In Readings in Groupware and ComputerSupported Cooperative Work: Assisting Human-Human Collaboration, Ed. R.M. Baecker. Morgan Kaufmann Publishers, Inc., San.

Beca, L. 1999. TANGO INTERACTIVE - A Collaborative Environment for the WorldWide Web (White Paper), Northeast Parallel Architectures Center, Syracuse University, Syracuse, New York.

Bennett, D.1995. Collaborative Spatial Decision Making for Ecosystem Management, Report from the Specialist Meeting on Collaborative Spatial Decision Making, Initiative 17, National Center for Geographic Information Analysis, UC Santa Barbara, September 17-21, 1995.

Brewer, I., MacEachren, A. M., Abdo, H., Gundrum, J., and Otto, G. 2000, Collaborative Geographic Visualization: Enabling shared understanding of environmental processes. IEEE Information Visualization Symposium, Salt Lake City Utah, Oct. 2000, pp. 137-141.

Carver, S. 1991, Integrating multi-criteria evaluation with geographical information systems. International Journal of Geographical Information Systems, 5(3), 321-339.

Churcher, N. and Churcher, C. 1999. RealTime Conferencing in GIS, Transactions in GIS, 1999, 3(1): 23-30.

Churcher, N. and Churcher, C. 1996. GROUPARC---A Collaborative Approach to GIS, Proceedings of 8th Annual Colloquium of the Spatial Information Research Centre, University of Otago, New Zealand, July 9-11, 1996, pp. 156-163

Cohen, P., McGee, D., Oviatt, S., Wu, L., Clow, J., King, R., Julier, S., and Rosenblum, L. 1999. Multimodal interaction for 2D and 3D environments. IEEE Computer Graphics and Applications, July/August.

Craig, W. J., and Elwood, S. A. 1998. How and why community groups use maps and geographic information. Cartography and Geographic Information Systems, 25(2), 95-104.

Densham, P. J., Armstrong, M. P., and Kemp, K. K. 1995. NCGIA Initiative 17 on Collaborative Spatial Decision making. Ellis C A, Gibbs S J and Rein G L 1991 Groupware: Some issues and experiences. Communications of the ACM 34: 38-58.

Densham, P. 1991 Spatial Decision Support Systems, in D.J. Maguire, M.F. Goodchild, and D.W. Rhind (Ed.) Geographical Information Systems: Principles and Applications, Longman, London, 403-412.

DeSanctis, G., and Poole, M. S. 1994. Capturing the complexity in advanced technology use: adaptive structuration theory. Organization Science, 5(2),121-147. 
Ellis, C., Gibbs, S., Rein, G.1991. Groupware Some Issues and Experiences, Communications of the ACM, Volume 34, No. 3, pp. 39-58, January 1991.

Evans, A., Kingston, R., and Ian, T. 1999. Web-based GIS to Enhance Public Democratic Involvement. Geocomp99 Conference Proceedings.

Faber, B. G., Small, L. R., and Wallace, W. W. 1997. Active response GIS: collaborative modeling for resource management. Paper presented at the ACSM/ASPRS, Seattle, Washington.

Faber B G, Knutson J, Watts R, Wallace W, Hautaluoma J E, and Wallace L. 1994. A groupware enabled GIS. In Heit M, Parker H D and Shortreid A (eds) GIS 94: GIS Applications in Natural Resources 2. Vancouver, 3-13.

Finley, D. 1997. A Collaborative GIS in a Distributed Work Environment. M.Eng. Report. Department of Geodesy and Geomatics Engineering University of New Brunswick, Fredericton, N. B., Canada.

Healey, P. 1996. The communicative turn in planning theory and its implications for spatial strategy formation. Environment and Planning B: Planning and Design, 23, 217-234.

Gutwin, Carl and Saul Greenberg 2001. A Descriptive Framework of Workspace Awareness for Real-Time Groupware. Computer Supported Cooperative Work.

Gutwin, Carl and Saul Greenberg 1999. The Effects of Workspace Awareness Support on the Usability of Real-Time Distributed Groupware. ACM Transactions on ComputerHuman Interaction 6(3): 243-281.

Hall, G.B., R.L. Bowerman and R.D. Feick. 1997. GIS-Based Decision Support Architecture And pplications For Developing Countries, South African Journal of Geo-Information, 17(3): 73-80.

Howard, D. 1998. Geographic information technologies and community planning: Spatial empowerment and public participation. Paper presented at the Project Varenius Meeting on Empowerment, Marginalization, and Public Participation GIS, Santa Barbara.

Idaho Corridor Planning Guidebook, 1998, http://www.itd.idaho.gov/planning/reports/corrplan/toc.html.

Li, S. 2003. Design and Development of an Internet Collaboration System to Support Distributed GIS Data Production Management. Ph.D. dissertation, Department of Geodesy and Geomatics Engineering Technical Report No. 219, University of New Brunswick, Fredericton, New Brunswick, Canada. 
Jackson, L.1999. Java Collaborative Technology Selections in NCSA Habanero, Proceedings of the 2nd International Conference on Concurrent Engineering in Construction, 25-27 August 1999, CIB Publication 236, Espoo, Finland, pp 37-46.

Jankowski, P. and Nyerges, T. 2001 a, Geographic Information Systems for Group Decision Making, Taylor and Francis, London.

Jankowski, P. and Nyerges, T. 2001 b, GIS-supported collaborative decision making: Results of an Experiment. Annals of the Association of American Geographers, 91(1), 48-70.

Jankowski, P., Nyerges, T. L., Smith, A., Moore, T. J., and Horvath, E. 1997. Spatial group choice: a SDSS tool for collaborative spatial decision making, International Journal of Geographical Information Science, Vol. 11, No. 6, pp. 577-602.

Jankowski, P. and Richard. 1994, Integration of GIS-based suitability analysis and multicriteria evaluation in a spatial decision support system for route selection. Environment and Planning, B, 21, 323-340.

Jones, R. M., Copas, C., V., and Edmonds, E. A. 1997. GIS support for distributed groupwork in regional planning. International Journal of Geographical Information Science, 11(1), 53-71.

MacEachren, A. M., Isaac Brewer, 2003. Developing a conceptual framework for visually-enabled geocollaboration. International Journal of Geographical Information Science 18(1): 1-34 (2004).

MacEachren, A. M., 2001. Cartography and GIS: Extending collaborative tools to support virtual teams. Progress in Human Geography, 25(3), 431-444

MacEachren, A. M., Brewer, I., and Steiner, E. 2001. Geovisualization to mediate collaborative work: tools to support different-place knowledge construction and decision making. Proceedings, $20^{\text {th }}$ International Cartographic Conference, Beijing, China, August 6-10, 2001, pp. 2533-2539.

MacEachren, A.M. 2000. Cartography and GIS: facilitating collaboration, Progress in Human Geography, vol. 24, pp. 445-456.

MacEachren, A. M., Edsall, R., Haug, D., Baxter, R., Otto, G., Masters, R., Fuhrmann, S., and Qian, L. 1999, Virtual environments for geographic visualization: Potential and challenges. Proceedings of the ACM Workshop on New Paradigms in Information Visualization and Manipulation, Kansas City, KS, Nov. 6, 1999 (also at: www.geovista.psu.edu/publications/NPIVM99/ammNPIVM.pdf), pp. 35-40.

Mateo, Calif. Dix, A., Beale, R. 1996. Remote Cooperation: CSCW Issues for Mobile and Teleworkers, Springer-Verlag London Limited, UK. 
McGee, D. R. and Cohen, P. R. 2001. Creating tangible interfaces by augmenting physical objects with multimodal language. 9th International Conference on HumanComputer Interaction, New Orleans, LA.

McGee, D. R., Cohen, P. R., and Wu, L. 2000, Something from nothing: Augmenting a paper based work practice via multimodal interaction. Proceedings of the ACM Designing Augmented Reality Environments DARE 2000, Helsinor, Denmark, pp. 71-80.

McGrath, J. E. 1984. Groups: Interaction and performance. Englewood Cliffs, NJ: Prentice-Hall.

Mosvick, R.K. and Nelson, R.B. 1987. We've Got to Start Meeting Like This: A Guide to Successful Meeting Management, Scott Foresman: Glenview, Ill.

N. Karacapilidis and C. 1997. Pappis: A framework for Group Decision Support Systems: Combining AI tools and OR techniques. European Journal of Operational Research, Special Issue on AI tools for Decision Support Systems, Vol. 103, No. 2, 1997, pp. 101-116.

Nyerges, T. L., and Jankowski, P. 1997. Enhanced adaptive structuration theory: A theory of GIS-supported collaborative decision making. Geographical Systems, 4(3), 225-259.

Nyerges, T., Barndt, M., and Brooks, K. 1997. Public participation geographic information systems. Paper presented at the ACSM/ASPRS, Seattle, Washington.

Nyerges, T. L., Montejano, R., Oshiro, C., and Dadswell, M. 1997, Group-based geographic information systems for transportation improvement site selection. Transportation Research C, 5(6), 349-369.

Obermeyer, N.J. 1998. The evolution of public participation GIS. Cartography and Geographic Information Systems 25, 65-66.

Oviatt, S., Angeli, A. D., and Kuhn, K. 1997, Integration and synchronization of input modes during multimodal human-computer interaction. Proceedings of the Conference on Human Factors in Computing Systems (CHI'97), pp. 415-422.

Pang, A. and Fernandez, D. 1995. REINAS instrumentation and visualization. Proceedings, OCEANS '95. MTS/IEEE. Challenges of Our Changing Global Environment, San Diego, Oct. 9-15, pp. 1892-1899.

Peng, Z., and D. Nebert, 1997. An Internet-Based GIS Data Access System. Journal of the Urban and Regional Information Systems Association 9: 20-30.

Podgorny, Marek, Walczak, Krzysztof, Warner, Dave, and C. Fox, Geoffrey, 1998. Internet Groupware Technologies - Past, Present, and Future, International Conference on Business Information Systems BIS 1998, Poznań, Poland; April 1998. 
Reitsma, R.F. 1996. Structure and support of water resources management and decision making, J. of Hydrology 177, 253-268.

Rhind D. 1997. The Framework for the World. New York, John Wiley and Sons (http://ncgia.ucsb.edu/research/i17/htmlpapers/golay/Golay.html): Santa Barbara, NCGIA.

Rinner, C. 2001. Argumentation maps: GIS-based discussion support for online planning. Environment and Planning B-Planning and Design, 28, 847-863.

Rinner, C. 1999. Argumentation Maps - GIS-based discussion support for online planning. $\quad \mathrm{PhD}$ Dissertation. University of Bonn. http://www.gmd.de/publications/research/1999/022/.

Rinner, C. 1997, Discussing plans via the World-Wide Web. 15th ECAADE, Vienna University of Technology.

Roche, S., and Humeau, J. B. 1999. GIS development and planning collaboration: A few examples from France.URISA Journal, 11(1).

Roseman, M. and Greenberg, S., 1992. GroupKit: A groupware toolkit for building realtime conferencing applications. In Proceedings of the ACM CSCW Conference on Computer Supported Cooperative Work, pp. 43-50, Toronto, Canada, November 1-4, ACM Press.

Scientific American 1990. Speciallssue on Communications, Computers, and Networks, 265 (3), September.

Shiffer, M. J. 1998. The evolution of public participation GIS. Cartography and Geographic Information Systems, 25(2), 89-94.

Shiffer, M. J. 1995. Geographic interaction in the city planing context: Beyond the multimedia prototype. In T. L. Nyerges and e. al. (Eds.), Cognitive Aspects of Human-Computer Interaction for Geographic Information Systems, pp. 295-310. Netherlands: Kluwer Academic Publishers.

Shiffer, M. J. 1993. Augmenting geographic information with collaborative multimedia technologies. Paper presented at the Auto-Carto 11, Minneapolis, MN, Oct. 30 - Nov. $1,1993$.

Shiffer, M. J. 1992. Towards a collaborative planning system. Environment and Planning B: Planning and Design, 19, 709-722.

Simon, H. and Associates 1992. Decision making and problem solving. In Decision making: Alternatives to Rational Choice Models, eds. M. Zey, pp. 32-53 Sage, Newbury Park, CA. 
Simon, H. 1976. From substantive to procedural rationality. In S. J. Latsis (Ed.), Method and Appraisal in Economics, pp. 129-148. London: Cambridge University Press

Smith, R. O'Shea, B., O'Malley, T., Scanlon, C., and Taylor, J. 1989. Preliminary Experiments with a Distributed, Multi-Media, Problem Solving Environment. In the proceedings of First European Conference on Computer Supported Cooperative Work, pp 19-34.

Sprague, R. 1980. A Framework for the Development of Decision Support Systems, MIS Quarterly, Vol. 4, No. 4.

Wood, J., Wright, H., and Brodlie, K. 1997. Collaborative visualization. Proceedings, IEEE Information Visualization '97, Pheoniz, Oct. 19-24, 1997, pp. 253-259.

Xiang, W. N., Gross, M., Fabos, J. G., and MacDougall, E. B. 1992. A fuzzy-group multicriteria decision making model and its application to land-use planning. Environment and Planning B, 19, 61-84.

Wood, J., Brodlie, K. and Wright, H. 1996. Visualization over the world wide web and its application to environmental data. IEEE Conference on Visualization, San Francisco, CA, 27 October to 1 November, 81-86.

Wood, J., Wright, H. and Brodlie, K. 1997. Collaborative visualization. Proceedings, IEEE Information Visualization ‘97, Phoenix, AZ, 19-24 October, 253-60.

Udell, J., Asthagiri, N., Tuvell, W. 2001. Peer-To-Peer: Harnessing the Power of Disruptive Technologies, O'Reilly and Associates.

Zhang, C. and M. Day. 2002. Development of a GIS-Based Spatial Decision Support System on the Internet for Conservation of Stone Forest Landscape in Lunan, China. UCGIS Summer Assembly 2002, Athens, Georgia, June 26-30, 2002.

Zwass, V. 1998. Foundations of Information Systems, Irwin McGraw-Hill, Boston, Massachusetts. 